\title{
1 Identification of novel avian and mammalian deltaviruses provides new insights
}

\section{2 into deltavirus evolution}

3

4 Masashi Iwamoto ${ }^{1,2}$, Yukino Shibata ${ }^{3}$, Junna Kawasaki ${ }^{4,5}$, Shohei Kojima ${ }^{4,6}$, Yung-Tsung

$5 \mathrm{Li}^{7}$, Shingo Iwami ${ }^{2}$, Masamichi Muramatsu ${ }^{1}$, Hui-Lin $\mathrm{Wu}^{7,8}, \mathrm{Kazuhiro} \mathrm{Wada}^{3}$, Keizo

6 Tomonaga ${ }^{4,5,9}$, Koichi Watashi ${ }^{1,10}$, Masayuki Horie ${ }^{4,11, *}$

$8 \quad{ }^{I}$ Department of Virology II, National Institute of Infectious Diseases, Tokyo, Japan

$9{ }^{2}$ Mathematical Biology Laboratory, Department of Biology, Faculty of Sciences, Kyushu University,

10 Fukuoka, Japan

$11{ }^{3}$ Graduate School of Life Science, Hokkaido University, Sapporo, Hokkaido, Japan

$12{ }^{4}$ Institute for Frontier Life and Medical Science, Kyoto University, Kyoto, Japan

$13{ }^{5}$ Department of Mammalian Regulatory Network, Graduate School of Biostudies, Kyoto University,

14 Kyoto, Japan

$15{ }^{6}$ Genome Immunobiology RIKEN Hakubi Research Team, RIKEN Cluster for Pioneering Research

$16{ }^{7}$ Hepatitis Research Center, National Taiwan University Hospital, Taipei, Taiwan

$17{ }^{8}$ Graduate Institute of Clinical Medicine, National Taiwan University College of Medicine

$18{ }^{9}$ Department of Molecular Virology, Graduate School of Medicine, Kyoto University, Kyoto, Japan

$19{ }^{10}$ Department of Applied Biological Sciences, Tokyo University of Science, Noda, Japan

$20 \quad{ }^{11}$ Hakubi Center for Advanced Research, Kyoto University

$22 *$ Corresponding author

23 Masayuki Horie, DVM, PhD

24 Hakubi Center for Advanced Research, Kyoto University

2553 Kawahara-cho, Shogo-in, Sakyo, Kyoto 606-8507, Japan 
bioRxiv preprint doi: https://doi.org/10.1101/2020.08.30.274571; this version posted October 18,2020 . The copyright holder for this preprint (which was not certified by peer review) is the author/funder, who has granted bioRxiv a license to display the preprint in perpetuity. It is made available under aCC-BY-NC-ND 4.0 International license.

26 Email: horie.masayuki.3m@kyoto-u.ac.jp

27 


\section{Abstract}

29 Hepatitis delta virus (HDV) is a satellite virus that requires hepadnavirus envelope

30 proteins for its transmission. Although recent studies identified HDV-related deltaviruses

31 in certain animals, the evolution of deltaviruses, such as the origin of HDV and the

32 mechanism of its coevolution with its helper viruses, is unknown, mainly because of the

33 phylogenetic gaps among deltaviruses. Here we identified novel deltaviruses of passerine

34 birds, woodchucks, and white-tailed deer by extensive database searches and molecular

35 surveillance. Phylogenetic and molecular epidemiological analyses suggest that HDV

36 originated from mammalian deltaviruses and the past interspecies transmission of

37 mammalian and passerine deltaviruses. Further, metaviromic and experimental analyses

38 suggest that the satellite-helper relationship between HDV and hepadnavirus was

39 established after the divergence of the HDV lineage from non-HDV mammalian

40 deltaviruses. Our findings enhance our understanding of deltavirus evolution, diversity,

41 and transmission, indicating the importance of further surveillance for deltaviruses. 


\section{Introduction}

43 Hepatitis delta virus (HDV) is the only member of the genus Deltavirus which is not

44 assigned to a family (1). The HDV genome is an approximately $1.7-\mathrm{kb}$ circular, negative

45 single-stranded RNA, harboring a single open reading frame (ORF) encoding the small

46 and large hepatitis delta antigens (S-HDAg, $24 \mathrm{kDa}$ and L-HDAg, $27 \mathrm{kDa}$ ) that are

47 translated from the same transcriptional unit via RNA-editing of the stop codon, which is

48 catalyzed by the host protein ADAR1 (2-5). The 19 amino acid residue extension of the

49 C-terminal region of L-HDAg contains a farnesylation site required to interact with

50 helper virus envelope proteins (6). The genome structure of HDV is unique in that it has

51 genomic and antigenomic ribozymes, which are essential for its replication $(7,8)$, and is

52 highly self-complementarity, generating a rod-like structure (9-11). Although HDV can

53 autonomously replicate, it requires an envelope protein of other "helper" viruses to

54 produce infectious virions. Hepatitis B virus (HBV) (family Hepadnaviridae) provides

55 the envelope proteins required for HDV transmission between humans (12).

56 Approximately 15-20 million people worldwide are estimated to be infected with HDV

57 among 350 million HBV carriers (13). Compared with mono-infection with HBV,

58 coinfection of HDV and HBV accelerates the pathogenic effects of $\mathrm{HBV}$, such as severe

59 or fulminant hepatitis and progression to hepatocellular carcinoma, through unknown

60 mechanisms (14).

61 The evolutionary origin of HDV presents an enigma. However, recent discoveries of

62 deltaviruses of vertebrate and invertebrate species (15-18), significantly changed our

63 understanding of deltavirus evolution. These non-HDV deltaviruses are distantly related

64 to HDV but may share the same origin because of their similarly structured circular RNA

65 genomes (approximately $1.7 \mathrm{~kb}$ ), which encode DAg-like proteins, possess ribozymes 
66 sequences, and are highly self-complementary (15-18). These findings provide clues to

67 the mechanism of deltavirus evolution. For example, a recent study hypothesizes that

68 mammalian deltaviruses codiverged with their host mammalian species (18). However,

69 the few known deltaviruses are highly divergent (15-18). Therefore, the phylogenetic

70 gaps between the deltaviruses must be filled through the identification of putative novel

71 deltaviruses.

72 The discoveries of non-HDV deltaviruses provides insights into the relationships

73 between deltaviruses and their helper viruses. Recently identified non-HDV deltaviruses

74 likely do not coinfect with hepadnaviruses, suggesting the presence of other helper

75 viruses (15-18). This hypothesis is supported by absence of a large isoform of DAg,

76 which is required for the interaction of HDV with the HBV envelope proteins, in rodent

77 deltavirus (18). Further, viral envelope proteins of reptarenavirus and hartmanivirus, but

78 not HBV, confer infectivity upon the snake deltavirus (19). These findings suggest that

79 hepadnaviruses do not serve as helper viruses for non-HDV deltaviruses and that the

80 deltavirus-hepadnavirus relationship is specific to the HDV lineage. However, the large

81 phylogenetic gap between HDV and the few other deltaviruses makes it difficult to assess

82 the hypothesis, raising the importance of further research.

83 In this study, to understand the evolution of deltaviruses, we analyzed publicly

84 available transcriptome data and found novel mammalian and avian deltaviruses. Our

85 phylogenetic analysis suggests that HDVs originated from non-HDV mammalian

86 deltaviruses and does not support the codiversification hypothesis of deltavirus and

87 mammalian evolution. Moreover, in silico and experimental analyses, together with

88 previous findings, suggest that the satellite-helper relationship between HDV and

89 hepadnavirus was established after the divergence of the novel non-HDV mammalian 
90 deltaviruses and the HDV lineage. Further, we present evidence for recent interfamily

91 transmission of deltaviruses among passerine birds. Our findings therefore provide novel

92 insights into the evolution of deltaviruses.

93 


\section{Results}

95 Identification of deltavirus-related sequences in avian and mammalian transcriptomes

96 We first assembled 46,359 RNA-seq data of birds and mammals. Using the resultant

97 contigs as queries, we identified five deltavirus-related contigs in the SRA data of birds

98 and mammals, including the zebra finch (Taeniopygia guttata), common canary (Serinus

99 canaria), Gouldian finch (Erythrura gouldiae), Eastern woodchuck (Marmota monax),

100 and white-tailed deer (Odocoileus virginianus). We named the deltavirus-like sequences

101 Taeniopygia guttata deltavirus (tgDeV), Serinus canaria-associated deltavirus (scDeV),

102 Erythrura gouldiae deltavirus (egDeV), Marmota monax deltavirus (mmDeV), and

103 Odocoileus virginianus (ovDeV), respectively (Table 1).

104 The amino acid sequences tlanslated from the contigs are $36.0 \%-66.7 \%$ identical to

105 those of the DAg proteins of known deltaviruses (Table 1, Supp Tables 1 and 2). The

$106 \operatorname{tgDeV}, \mathrm{mmDeV}$, and ovDeV contigs, which comprise approximately 1,700 nucleotides,

107 encode one ORF with a sequence similar to those of DAg genes of known deltaviruses

108 (Fig 1a, Table 1, Supp Tables 1 and 2). In contrast, the contigs scDeV and egDeV are 761

109 and 596 nucleotides in length, respectively (Fig. 1b, Supp Tables 1 and 2). Note that the

110 nucleotide sequences of $\operatorname{tgDeV}$ and $\mathrm{egDeV}$ are $97.7 \%$ identical, and we therefore

111 analyzed $\operatorname{tgDeV}$ instead of $\operatorname{tgDeV}$ and $\mathrm{egDeV}$.

112

113 Genome structures of novel avian and mammalian deltaviruses

114 The three contigs (tgDeV, mmDeV, and ovDeV) are almost identical in length to the

115 full-length genomes of known deltaviruses. We therefore checked for potential circularity

116 of the contigs. Dot-plot analyses revealed that each of both ends of these three contigs is

117 identical (Supp Fig. 1), suggesting that the contigs were derived from circular RNAs. We 
118 further mapped the original RNA-seq data to the corresponding circularized contigs using

119 the Geneious mapper, revealing that some of the reads properly spanned the junctions

120 (data not shown), indicating that these contigs are derived from circular RNAs. Therefore,

121 we designated the resultant circular contigs of $\operatorname{tgDeV}, \mathrm{mmDeV}$, and ovDeV as full-length

122 novel deltavirus genomes (1,706, 1,712, and 1,690 nucleotides, respectively) (Fig. 1a).

123 These novel genomes are characterized by high self-complementarity, genomic and

124 antigenomic ribozymes, and poly(A) signals, which are conserved among known

125 deltaviral genomes (Fig. 1 and Supp Table 2) (15-18). Further, the predicted secondary

126 structures of the ribozymes are highly similar to those of HDV as well as those of other

127 deltaviruses (Supp Fig. 2).

129 Characterization of DAg proteins encoded by the novel deltaviruses

130 We next characterized the putative DAg proteins encoded by the novel deltaviruses. Most

131 of their biochemical features, biologically relevant amino acid residues, and functional

132 domains (15-18) are conserved among the DAg proteins (Fig. 2a). The isoelectric points

133 of DAg proteins from the novel deltaviruses range from 10.35 to 10.63 (Supp Table 2),

134 which are nearly identical to those of known deltaviruses. All the post-translational

135 modification sites in HDAg are conserved among those of all DAg proteins of the novel

136 deltaviruses, except for the serine phosphorylation site on scDeV-DAg (Fig. 2a). The

137 NLS is conserved among the DAg proteins, although location of the predicted NLS of

138 scDeV DAg protein differs (Fig. 2a).

139 We next investigated whether the novel deltaviruses utilize A-to-I RNA-editing. To

140 answer this question, we mapped short reads of the SRA data, which we initially used to

141 detect the deltaviruses, to identify the nucleotide variations among the stop codons. We 
142 found a potential RNA-editing site within the stop codon of the ovDeV-DAg gene, in

143 which there was $0.4 \%$ nucleotide variation (5 of 1160 reads) at the second nucleotide

144 position of the stop codon (U므), all of which were $\mathrm{G}$ instead of the consensus

145 nucleotide A (Fig. 2b). The quality scores of the five $\mathrm{G}$ variants ranged from 35 to 41

146 (Supp Fig. 3), which likely exclude the possibility of a sequencing error. This variation

147 may be explained by A-to-I editing by ADAR1, as known for HDV (2). However,

148 possible RNA-editing generates a protein two amino acid residues longer because of a

149 stop codon immediately downstream (Fig. 2c). Further, the C-terminal farnesylation

150 motif (CXXQ) required for the interaction with hepadnaviral envelope proteins (6) was

151 absent from the longer product. These observations suggest that even if RNA-editing

152 occurs, the resultant gene product does not contribute to the interaction with hepadnaviral

153 envelope proteins. Further, we were unable to identify nucleotide variations of the

154 mapped reads at the stop codons in the genomes of $\operatorname{tgDeV}$ and $\mathrm{mmDeV}$ (data not shown).

The novel deltaviruses potentially replicate in their hosts

157 To determine whether the novel deltaviruses potentially replicate in their respective,

158 putative host species, we evaluated the mapping pattern of viral reads described above.

159 We found that the read depths of the predicted transcribed regions (the DAg coding

160 regions to poly-A signals) were much greater than those of the other genomic regions (Fig.

1613 ), indicating that most viral reads were derived from viral mRNAs. These findings

162 suggest that the novel deltaviruses replicate in their hosts.

163 The mapping pattern on tgDeV differed slightly from the others. Specifically,

164 although the read depth of the DAg ORF region was higher, the reads represented only

$16580 \%$ of the ORF (Fig. 3a). This trend was apparent in another tgDeV-positive RNA-seq 
166 data (Supp Fig. 4). However, it is not clear whether this is attributed to an artifact or

167 actually reflects the transcription pattern of $\operatorname{tgDeV}$.

168

169 Transmission of tgDeV-and tgDeV-like viruses among passerine birds

170 We next investigated whether the novel deltaviruses are transmitted among animal

171 populations. We first analyzed tgDeV infections in birds using RNA-seq data (Table 2

172 and Supp Table 3). Among 6453 SRA data, $\operatorname{tgDeV}$-derived reads were identified in 34

173 SRAs, including the SRAs in which tgDeV and egDeV were initially detected. The 34

174 tgDeV-positive SRA data were obtained from tissues such as blood, kidney, and muscles,

175 suggesting broad tropism and viremia, or systemic infection, or both, with tgDeV.

176 Further, tgDeV sequences were detected in several bird species such as the

177 black-headed bunting (Emberiza melanocephala) and yellow-bellied tit (Pardaliparus

178 venustulus). All tgDeV-positive bird species belong to the order Passeriformes. These

179 tgDeV-positive SRA data are included in the nine BioProjects deposited by independent

180 researchers, and thus the birds were likely from different sources. Further, the

181 tgDeV-positive sample in SRR9899549 (BioSample accession: SAMN12493457) is

182 derived from a black-headed bunting caught in the wild. These data suggest that tgDeV

183 (or tgDeV-like viruses) circulate among diverse passerine birds, even in the wild.

184 During the above analysis, we found that SRA data from the yellow-bellied tit

185 (SRR7244693 and SRR7244695-SRR7244698) contain many reads mapped to the

$186 \operatorname{tgDeV}$ genome. Therefore, we extracted the mapped reads of SRA data and performed de

187 novo assembly. We obtained a 1707-nt circular complete genome sequence, which we

188 designated pvDeV. The pvDeV nucleotide sequence is $98.2 \%$ identical to that of $\operatorname{tgDeV}$,

189 and the properties of its DAg protein sequence are similar to those of $\operatorname{tgDeV}$ DAg (Supp 
190 Fig. 5).

191 We next employed real-time RT-PCR to further evaluate potential deltavirus

192 infections of passerine birds. We analyzed 30 and 5 whole-blood samples from zebra and

193 Bengalese finches (Lonchura striata var. domestica), respectively, and found that one

194 Bengalese finch was positive for real-time RT-PCR test. To exclude the possibility of

195 contamination of a plasmid used as a control for real-time PCR, we performed RT-PCR

196 using a primer set that distinguishes viral from plasmid amplicons (Figs. 4a and b). We

197 obtained a band of the expected size only from the cDNA sample (Fig. 4c), revealing that

198 the bird was truly positive for a tgDeV-like virus. Therefore, we named this virus lsDeV,

199 and further analysis revealed that its full-length genome nucleotide sequence (1708 nt) is

$20098.2 \%$ and $98.4 \%$ identical to those of $\operatorname{tgDeV}$ and $\mathrm{pvDeV}$, respectively. Moreover, its

201 genome features are almost identical to those of $\operatorname{tgDeV}$ and pvDeV (Supp Fig. 5).

Evidence for recent interfamily transmission of deltaviruses among passerine birds

204 We found that the sequence similarities among the passerine deltaviruses (tgDeV, pvDeV,

205 and ls DeV) (Fig. 4d) were not consistent with evolutionary codivergence. According to

206 the TimeTree (20), deltavirus-positive passerine birds diverged approximately 44 million

207 years ago (Fig. 4e and Supp Fig. 6). Considering the rapid evolutionary rates of HDVs

208 (approximately $10^{-3}$ substitutions per site per year) (21-23), it is unlikely that these

209 viruses codiverged with their hosts. Most likely, interfamily transmission occurred

210 relatively recently among passerine birds.

\section{Transmission of mmDeV among woodchucks}

213 We similarly analyzed mmDeV infections using SRA data for the order Rodentia, other 
214 than mice (Mus musculus) and rats (Rattus norvegicus). Our analysis of 4776 SRA

215 datasets detected mmDeV reads in 20 SRA data of seven woodchucks (Table 2 and Supp

216 Table 3). Although these mmDeV-positive SRA data were contributed by the same

217 research group, the animals were apparently obtained at different times $(24,25)$,

218 suggesting that mmDeV was transmitted among woodchucks. The mmDeV-positive SRA

219 data are derived from samples of liver or peripheral blood mononuclear cells.

220 We next used real-time RT-PCR to analyze 81 woodchuck samples (liver, $\mathrm{n}=43$;

221 serum, $\mathrm{n}=38$ ). However, $\mathrm{mmDeV}$ was undetectable (data not shown), which may be

222 explained by the absence of $\mathrm{mmDeV}$ infection or clearance, low level of infection, or

223 both.

\section{No evidence of transmission of other deltaviruses}

226 We next focused on ovDeV and $\mathrm{scDeV}$ sequences of ruminant animals and passerine

227 birds, respectively (Table 2 and Supp Table 3). We detected ovDeV-derived reads only in

228 five SRA data. The SRA data were obtained from brain, muscle, testis, pedicles, and

229 antlers, suggesting systemic infection, viremia, or both. However, we were unable to

230 determine if these samples were derived from multiple individuals. We detected

231 scDeV-derived reads only from the SRA data in which the virus was initially detected. We

232 therefore were unable to provide evidence for the transmission of ovDeV and $\mathrm{scDeV}$ in

233 their host animals.

235 Phylogenetic relationships among deltaviruses

236 To decipher the evolutionary relationships among deltaviruses, we conducted a

237 phylogenetic analysis using known and the novel deltavirus sequences discovered here. 
238 We did not include sequences of recently identified fish, toad, newt, termite, and

239 duck-associated deltaviruses because they share very low amino acid sequence identities

240 with the novel deltaviruses as well as with HDVs (Fig. 5a), which may reduce the

241 accuracy of tree (16). We further excluded $\mathrm{scDeV}$ for this reason, and we did not include

$242 \operatorname{tgDeV}$-like viruses, because their sequences are nearly identical to that of $\operatorname{tgDeV}$. The

243 reconstructed tree shows that the newly identified $\operatorname{tgDeV}$ forms a strongly supported

244 cluster with snake $\mathrm{DeV}$ and rodent $\mathrm{DeV}$, although they are distantly related to each other

245 (Fig. 5b). Note that $\mathrm{mmDeV}$ and ovDeV are more closely related to HDVs than the other

246 deltaviruses.

248 Candidate helper viruses

249 To gain insights into helper viruses of the novel deltaviruses, we first analyzed the

250 coexisting viruses in the SRA data using BLASTx. Note that we omitted experimental

251 woodchuck hepatitis virus (WHV) infections associated with the mmDeV-positive

252 woodchuck-derived SRA data (SRR2136864 to SRR2136999). We also excluded viruses

253 that infect invertebrates and endogenous retroviruses as well. These analyses reveal that

254 polyomavirus, bornavirus, and circovirus sequences are present in the deltavirus-positive

255 SRA data of passerine birds (Table 3 and Supp Table 4). Further, we detected contigs with

$25698 \%-100 \%$ identities to human viruses (human mastadenovirus or mammalian

257 rubulavirus 5) (Supp Table 4), although these may represent contamination, index

258 hopping, or both. Among these viruses, only the genome of bornavirus encodes an

259 envelope protein. Note that $\mathrm{scDeV}$ and bornavirus-positive SRA data was obtained from

260 pooled samples (SAMN04260514), and we therefore were unable to determine whether

261 scDeV and canary bornavirus 3 infected the same individual. 
262 We next cross-referenced the mmDeV reads and the metadata, which also provide

263 insights into the mmDeV helper virus. Among $20 \mathrm{mmDeV}$-positive SRA data, 18 were

264 obtained from animals experimentally infected with the hepadnavirus WHV, which was

265 experimentally shown to serve as a helper virus for $\operatorname{HDV}(26,27)$. However, the other

266 two SRA data (SRR437934 and SRR437938) were derived from animals negative for

267 antibodies against WHV as well as WHV DNA (24). These observations suggest that

$268 \mathrm{mmDeV}$ was transmitted to the two animals without WHV and that WHV therefore was

269 not the helper virus for mmDeV that infected these two individuals.

270

271 Replication of tgDeV and mmDeV in human and woodchuck cell lines

272 To investigate whether the novel deltavirus sequences are replicable or not, we performed

273 transfection-based assays in cell culture systems. We constructed plasmid expression

274 vectors harboring the minus-strand genome of the $\operatorname{tgDeV}$ or $\mathrm{mmDeV}$ dimer sequence

275 under the transcriptional control of the CMV promoter (see Materials and Methods). We

276 first determined if the replication initiated by transfecting these plasmids. These plasmids

277 express the minus-strand genome and therefore DAg protein is expressed if the viral

278 genome replicates (19). We transfected the plasmid vectors into Huh7 human hepatic

279 cells and WCH-17 woodchuck hepatic cells and used western blotting (Figs. 6a and b)

280 and immunofluorescence assay (IFA) (Figs. 6c-f) to detect the expression of DAg

281 proteins. Western blotting detected the expected bands (approximately $22 \mathrm{kDa}$ ) only in

282 transfected cells (Figs. 6a and b). Note that a single specific band was detected in lysates

283 prepared from each cell type, suggesting that $\operatorname{tgDeV}$ and $\mathrm{mmDeV}$ expressed only one

284 DAg isoform. Consistent with the above results, specific signals were observed only in

285 the transfected cells in IFA (Figs. 6c and d, red signals). Together, these data suggest that 
286 the tgDeV and $\mathrm{mmDeV}$ initiated replication from the constructed plasmids in the cell

287 culture system.

288 The DAg proteins predominantly localized to the nucleus 2 days post-transfection

289 (Figs. 6e and f). Interestingly, large viral speckles were observed in the nucleus, similar to

290 those detected in cells infected with $\operatorname{HDV}(28,29)$. These results suggest that $\operatorname{tgDeV}$ and

291 mmDeV employ a nuclear replication strategy similar to that used by HDV.

HBV envelope proteins do not contribute to the production of infectious tgDeV and

$m m D e V$

295 As described in the above section "Candidate helper viruses", there is no evidence of

296 coinfections of hepadnaviruses with $\operatorname{tgDeV}$ or $\mathrm{mmDeV}$. However, this does not

297 necessarily mean hepadnaviruses do not serve as helper viruses for the novel deltaviruses.

298 To determine whether tgDeV or $\mathrm{mmDeV}$ utilize the $\mathrm{HBV}$ envelope proteins (HBs), we

299 transfected the deltavirus expression plasmids together with an HBs expression vector or

300 the cognate empty vector into Huh7 cells. The culture supernatants were incubated with

301 HepG2-NTCP cells, which are susceptible to HBs-dependent HDV infection (30, 31).

302 HDV served as a control to monitor HBs-dependent virus release and subsequent cell

303 entry. Viral RNA was undetected in supernatants of cells that did not express HBs (Fig.

304 7a). In contrast, cotransfection of the HBs plasmid released large amounts of HDV RNA

305 into the supernatant, consistent with published data (32), whereas $\operatorname{tgDeV}$ or $\mathrm{mmDeV}$

306 RNA was undetectable (Fig. 7a).

307 We next measured the amounts of viral RNA and detected DAg protein in

308 HepG2-NTCP cells 7 days after incubation with the supernatants. HDV RNA and DAg

309 protein were highly expressed in the infected cells (Fig. 7b and c). HDV infection was 
310 inhibited by the preS1 peptide (Myrcludex B), which was shown to inhibit

311 HBs-dependent HDV infection (33). These indicate that HDV infection is indeed

312 mediated by the HBs. On the other hand, $\operatorname{tgDeV}$ and mmDeV RNA or DAg protein was

313 undetectable, suggesting that HBs do not contribute to the production of infectious tgDeV

314 or mmDeV. 


\section{Discussion}

316 Important aspects of the evolution of deltaviruses are unknown, such as the origin of

317 HDV and the coevolution of deltaviruses and their helper viruses, mainly because few

318 deltaviruses are known, and they are highly genetically divergent (15-18). Therefore, the

319 resulting large gaps in the deltavirus phylogenetic tree create a formidable obstacle to

320 understanding deltavirus evolution. Here we identified five complete genomes of novel

321 deltaviruses from birds and mammals (Fig. 1 and Supp Fig. 5), which partially fill these

322 phylogenetic gaps (Fig. 5b). Moreover, our present findings reveal that the evolution of

323 deltaviruses is much more complicated than previously thought. For example, one

324 hypothesis states that mammalian deltaviruses codiverged with their mammalian hosts

325 (18). However, our phylogenetic analysis shows that the tree topology of mammalian

326 deltaviruses is incongruent with their hosts'. For example, ovDeV, which we detected in

327 deer, is most closely related to human HDV (Fig. 5b). Further, the distantly related

$328 \mathrm{mmDeV}$ and rodent DeV (detected in Proechimys semispinosus (18)) were detected in

329 rodent species. These data suggest that deltaviruses were transmitted among mammalian

330 species and did not always codiverge with their hosts. Moreover, we discovered recent

331 interfamily transmission of passerine deltaviruses (tgDeV and its relatives) (Fig. 4e).

332 Therefore, avian and mammalian deltaviruses may have, at least partially, evolved by

333 interspecies transmission.

334 Our present phylogenetic analysis also gives insights into the origin of human HDVs.

335 As described above, ovDeV and $\mathrm{mmDeV}$ are close relatives of human HDVs, suggesting

336 that HDVs arose from other mammalian deltaviruses. Recent studies on the phylogeny of

337 bat deltaviruses support our findings and conclusions (34, 35) (Supp Fig. 7). However, we

338 were unable to exclude the possibility of infection of animal lineages apart from 
339 mammals with unknown deltaviruses phylogenetically located between those viral

340 lineages. Further investigations are required for a better understanding of the deltavirus

341 evolution.

342 There is a paucity of knowledge about helper viruses for non-HDV deltaviruses,

343 other than the snake deltavirus (19), although evidence indicates that hepadnaviruses may

344 not serve as helper viruses for the novel deltaviruses discovered here, as suggested for

345 other non-HDV deltaviruses (15-18). Here we only detected bornavirus, circovirus, and

346 polyomavirus, but not hepadnavirus sequences in association with deltavirus-positive

347 SRA data (Table 3). Further, mmDeV was detected in two woodchuck individuals that

348 were demonstrated to be negative for WHV (Table 2 and Supp Table 3). Moreover, we

349 found that HBs did not contribute to the formation of infectious $\operatorname{tg} \mathrm{DeV}$ and $\mathrm{mmDeV}$ in

350 cell culture experiments. These observations suggest that hepadnaviruses do not serve as

351 helper viruses for the novel non-HDV deltaviruses detected here. Further, we were

352 unable to demonstrate that the deltaviruses identified here express proteins similar to the

353 L-HDAg protein (Figs. 6a and b), which is expressed via RNA-editing and is essential for

354 HDV to interact with HBs $(2,37)$. Although RNA-editing may alter the stop codon of

355 ovDeV DAg, this does not lead to the expression of a large isoform of DAg (L-DAg)

356 protein containing a farnesylation site (Figs. 2b and c). The lack of L-DAg expression

357 was also observed in rodent deltaviruses (18). Therefore, L-DAg expression phenotype

358 may have been acquired after the divergence of ovDeV and the HDV lineage (Fig. 5b).

359 Among the coexisting viruses, only bornavirus produces an envelope glycoprotein

360 (G protein), which might be used by non-HDV deltaviruses to produce infectious virions.

361 Indeed, snake deltavirus utilizes the envelope proteins of reptarenaviruses and

362 hartmaniviruses to produce infectious particles (19). Furthre, HDV forms infectious 
363 virions using envelope proteins in vitro of RNA viruses such as vesiculovirus and

364 hepacivirus (38). Therefore, the bornavirus G protein might envelop non-HDV

365 deltaviruses.

366 In contrast, the coexisting viruses, circoviruses and polyomaviruses, are

367 nonenveloped. Therefore, it is unlikely that these viruses can serve as helper viruses for

368 the deltaviruses. However, we cannot exclude the possibility that these viral capsid

369 proteins might contribute to the transmissibility of deltaviruses through unknown

370 mechanisms. Additionally, virus-derived sequences in host genomes, called endogenous

371 viral elements (EVEs), might mediate the formation of infectious particles. Here detected

372 the expression of retroviral EVEs in certain deltavirus-positive SRA data (data not

373 shown). Although HDVs do not use retroviral envelope proteins (38), non-HDV

374 deltaviruses might utilize strategies distinct from those employed by HDVs. Alternatively,

375 non-HDV deltaviruses may not require helper viruses and utilize extracellular vesicles for

376 transmission. Further biological experiments, together with molecular surveillance, are

377 therefore required to understand the satellite-helper relationships of deltaviruses.

378 Here we show that the sequences of $\operatorname{tgDeV}$ and tgDeV-like viruses, such as pvDeV

379 and lsDeV, are relatively closely related to known vertebrate deltaviruses (Fig. 5b).

380 Although a previous study found a deltavirus from duck, this duck-associated virus was

381 detected in oropharyngeal/cloacal swabs and is distantly related to vertebrate deltaviruses,

382 suggesting the possibility of its dietary origin $(15,18)$. This may be true for $\mathrm{scDeV}$

383 studied here. $\mathrm{scDeV}$ was detected in skin (Table 1). Although $\mathrm{scDeV}$ was excluded from

384 our phylogenetic analysis, the amino acid identities between the DAg protein of scDeV

385 with other vertebrate deltaviruses range from $32.7 \%-39.5 \%$ (Fig. 5a). Therefore, scDeV

386 may be derived from contaminants, which should be addressed in the future. In contrast, 
$387 \operatorname{tgDeV}$ and tgDeV-like viruses were detected in tissues such as the spleen and muscles

388 (Table 2), suggesting that $\operatorname{tgDeV}$ and tgDeV-like viruses are authentic avian deltaviruses.

389 Here we show that certain novel deltaviruses are transmitted among animal

390 populations (Table 2 and Supp Table 3). Note that few reads were mapped to the virus

391 genomes in some SRA data, which may be attributed to index hopping (39-43) from SRA

392 data containing numerous deltavirus-derived reads. Therefore, these data should be

393 interpreted with caution. Nevertheless, our conclusions are not affected, because they are

394 supported by robust data (Table 2). For data in which index hopping has possibly

395 occurred, further analyses are needed to confirm deltavirus infections.

396 Our present analysis provides virological insights into important characteristics of

397 deltavirus infections, such as tissue and host tropism. For example, infections with tgDeV

398 (and tgDeV-like viruses), $\mathrm{mmDeV}$, and ovDeV were not limited to the liver and were

399 detected in at least two different tissues (Table 2). These observations are consistent with

400 those of previous studies that non-HDV deltaviruses in multiple organs and blood and

401 that they replicate in numerous cell types $(18,19)$. Therefore non-HDV deltaviruses may

402 infect diverse tissues and cause systemic infection, viremia, or both. Furthre, $\operatorname{tgDeV}$ and

$403 \mathrm{mmDeV}$ replicated in human and woodchuck cells (Fig. 6), which is consistent with the

404 ability of the snake deltavirus to replicate in mammalian cells (19). These observations

405 suggest that the host range of deltaviruses is broad and that the helper viruses of

406 non-HDV deltaviruses may be the determinants of host range.

407 Our analyses further suggest that tgDeV and $\mathrm{mmDeV}$ are sensitive to host immune

408 responses. We cross-referenced tgDeV reads and metadata and made an intriguing

409 finding that may contribute to the virus-host interaction. BioProject PRJNA297576

410 contains 12 RNA-seq data for six zebra finches (44). Interestingly, the tgDeV reads were 
411 almost exclusively detected in birds treated with testosterone vs the controls (Supp Fig.

412 8a and Supp Table 5). Therefore, the immunosuppressive effects of testosterone (45) may

413 increase the transcription or replication of $\operatorname{tgDeV}$, or both, to enable detection using

414 RNA-seq. Furthre, when we cross-referenced the mmDeV reads and the metadata, we

415 found that 18 of $20 \mathrm{mmDeV}$-positive SRA data derived from five individuals were

416 acquired through an experiment lasting 27 weeks (PRJNA291589) (25). Among 18 SRA

417 data, those of one individual (ID 1008) provide insights into $\mathrm{mmDeV}$ infection as

418 follows: At first ( -3 weeks), mapped reads were not detected, although the proportion of

419 mapped reads were highest at one week and then drastically decreased (Supp Fig. 8b and

420 Supp Table 6). Interestingly, a previous study suggested that the host's immune response

421 can clear rodent deltaviruses (18). Our present observations together with this previous

422 finding, suggest that the host immune response suppresses and then clear deltavirus

423 infections. This may explain the low prevalence of RT-PCR-positive samples of

424 woodchucks and passerine birds as described in the Results (sections "Circulation of

$425 \mathrm{tgDeV}$ and tgDeV-like viruses among passerine birds" and "Circulation of mmDeV in

426 woodchucks"). Note that latent or low levels of persistent deltavirus infections may occur.

427 Indeed, snake deltavirus establishes a persistent infection in a cell culture system (19).

428 Therefore, deltaviruses might persistently infect host cells with a low level of virus

429 replication, and some stimulations, such as immunosuppression, may trigger robust virus

430 replication. Further studies are therefore required to understand the interactions between 431 deltaviruses and their hosts.

432 Together, our present data contribute to a deeper understanding of the evolution of

433 deltaviruses and suggest the presence of undiscovered deltaviruses that infect diverse

434 animal species. Further investigations will provide further insights into deltavirus 
bioRxiv preprint doi: https://doi.org/10.1101/2020.08.30.274571; this version posted October 18, 2020. The copyright holder for this preprint (which was not certified by peer review) is the author/funder, who has granted bioRxiv a license to display the preprint in perpetuity. It is made available under aCC-BY-NC-ND 4.0 International license.

\section{5 evolution.}

436 


\section{Materials and methods}

\section{Detection of deltaviruses from publicly available transcriptome data}

439 Paired-end, RNA-seq data from birds and mammals were downloaded from NCBI SRA

440 (46). The SRA accession numbers used in this study are listed in Supplementary material.

441 The downloaded SRA files were dumped using pfastq-dump (DOI:

442 10.5281/zenodo.2590842; https://github.com/inutano/pfastq-dump), and then

443 preprocessed using fastp 0.20 .0 (47). If genome data of either the same species or the

444 same genus were available, the preprocessed reads were mapped to the corresponding

445 genome sequences (the genome information is available upon request) by HISAT2 2.1.0

446 (48), and then unmapped paired-end reads were extracted using SAMtools 1.9 (49) and

447 Picard 2.20.4 (http://broadinstitute.github.io/picard/). The extracted unmapped reads

448 were used for de novo assembly. If genome data were unavailable, the preprocessed reads

449 were directly used for de novo assembly. De novo assembly was conducted using SPAdes

450 (50) and/or metaSPAdes (51) 3.13 .0 with k-mer of $21,33,55,77$, and 99 . The resultant

451 contigs were clustered by cd-hit-est $4.8 .1(52,53)$ with a threshold of 0.95 . Finally, the

452 clustered contigs $\geq 500 \mathrm{nt}$ were extracted by SeqKit 0.9.0 (54), and they were used for the

453 downstream analyses.

454 Two-step sequence similarity searches were performed to identify RNA virus-like

455 sequences. First, BLASTx searches were performed against a custom database for RNA

456 viruses using the obtained contigs as queries employing BLAST+2.9.0 (55) with the

457 following options:-word_size 2 , -evalue $1 \mathrm{e}^{-3}$, max_target_seqs 1 . The custom database of

458 RNA viruses consisted of clustered sequences (by cd-hit 4.8.1 with a threshold of 0.98 )

459 from viruses of the realm Riboviria in the NCBI GenBank (the sequences were

460 downloaded on June 2, 2019) (46). Next, the query sequences with viral hits were 
461 subjected to second BLASTx analyses, which were performed against the NCBI nr

462 database. Finally, the second blast hits with the best hit against deltaviruses were regarded

463 as deltavirus-like agents, and they were used for detailed analyses.

\section{Confirmation of circularities of deltavirus contigs}

466 Self dot-plot analyses of linear deltavirus contigs were conducted using the YASS online

467 web server (29). Based on the analysis, the contigs were manually circularized using

468 Geneious 11.1.5 (https://www.geneious.com). Further confirmation of the circularities of

469 deltavirus contigs was obtained by mapping short reads to circular deltavirus contigs

470 using Geneious software as follows. The reads used for the de novo assembly were first

471 imported to Geneious, after which they were mapped to the circular contigs using the

472 Geneious mapper. The mapped reads across the circularized boundaries were confirmed 473 manually.

476 We used the mapped reads obtained by the analyses described above to detect possible

477 RNA-editing at the stop codons of DAg genes of deltaviruses. We analyzed the

478 nucleotide variations (presence of variations, variant nucleotide(s), and variant

479 frequency) of mapped reads at each of the stop codons of newly identified deltaviruses

480 using the "Find Variation/SNPs" function in Geneious. We used a custom Python script to

481 visualize base quality scores of the NGS reads mapped at the second nucleotide of the

482 stop codon genome. The codes are available at following URL:

483 https://github.com/shohei-kojima/iwamoto_et_al_2020. 
486 DAg ORFs were detected by the "Find ORFs" function in Geneious with a threshold of

487500 nucleotides. Poly(A) signals were manually detected. Putative ribozyme sequences

488 were identified using nucleotide sequence alignment with other deltaviruses. Ribozyme

489 structures were first inferred using the TT2NE webserver (56), and the obtained data were

490 then visualized using the PsudoViewer3 web server (57). We used the visualized data as

491 guides to draw ribozyme structures.

492 The self-complementarities of deltavirus-like contigs were analyzed using the Mfold

493 web server (58). Coiled-coil domains and nuclear export signals (NLSs) were predicted

494 using DeepCoil (59) and NLS mapper

495 (http://nls-mapper.iab.keio.ac.jp/cgi-bin/NLS_Mapper_form.cgi) web servers,

496 respectively.

497

498

Short read mapping for detection of deltavirus infection

499 To detect deltavirus-derived reads in publicly available RNA-seq data, short reads were

500 mapped to deltavirus genomes and then the numbers of mapped reads were counted as

501 follows. SRA files were downloaded from NCBI, dumped, and preprocessed following

502 the procedure described above. The preprocessed reads were then mapped to linearized

503 deltavirus contigs by HISAT2 with the default setting. SAM tools were used to extract the

504 mapped BAM files from the resultant BAM files, and the mapped read numbers were

505 counted using BamTools 2.5.1 (60).

506

507 Recovery of a deltavirus genome from RNA-seq data of Pardaliparus venustulus

508 Mapped reads obtained from SRR7244693, SRR7244695, SRR7244696, SRR7244697, 
509 and SRR7244698 in the above analysis (section Short read mapping for detection of

510 deltavirus infection) were extracted by Geneious. All the extracted reads were

511 co-assembled using Geneious Assembler with the circular contig assembly function. The

512 obtained circular contigs were characterized as described previously.

514 Animals and samples

515 Zebra finches $(n=30)$ and Bengalese finches $(n=5)$ were obtained from breeding

516 colonies at Wada lab, Hokkaido University. The founder birds were originally obtained

517 from local breeders in Japan. Five to twelve birds were kept together in cages in an aviary

518 and were exposed to a 13:11 light-dark cycle. Blood samples were collected from the

519 wing vein using $30 \mathrm{G} \times 8 \mathrm{~mm}$ syringe needles (Becton Dickinson; Franklin Lakes, NJ,

520 USA). Each blood sample was diluted 1.5 times with PBS, frozen immediately on dry ice

521 after collection, and maintained at $-80^{\circ} \mathrm{C}$ until further requirement. These experiments

522 were conducted under the guidelines and with the approval of the Committee on Animal

523 Experiments of Hokkaido University. These guidelines are based on the national

524 regulations for animal welfare in Japan (Law for the Humane Treatment and

525 Management of Animals with partial amendment No.105, 2011).

526 Woodchucks (Marmota monax) were purchased from Northeastern Wildlife

527 (Harrison, ID, USA) and kept at the Laboratory Animal Center, National Taiwan

528 University College of Medicine. At three days of age, the animal supplier infected

529 captive-born woodchucks with WHV from the same infectious pool. Wild-caught

530 woodchucks were infected naturally and live trapped. Serum samples were collected

531 from the woodchucks periodically via the femoral vein by means of venipuncture. Liver

532 tissues of woodchucks were obtained at autopsy, snap-frozen in liquid nitrogen, and 
533 stored at $-80^{\circ} \mathrm{C}$ until RNA extraction. This study used liver tissues from 10 wild-caught

534 and 33 captive-born woodchucks and serum samples from 33 wild-caught and five

535 captive-born woodchucks. In this study, all the experimental procedures involving

536 woodchucks were performed under protocols approved by the Institutional Animal Care

537 and Use Committee of National Taiwan University College of Medicine.

539 Real-time and Endpoint RT-PCR detection of deltaviruses from animal specimens

540 Total RNAs were isolated from the whole blood samples from zebra finches and serum

541 samples from woodchucks using Quick RNA Viral Kit (Zymo Research; Irvine, CA,

542 USA). The obtained RNA samples were stored at $-80^{\circ} \mathrm{C}$ until further requirement. Total

543 RNAs were also extracted from $50 \mathrm{mg}$ of the woodchuck liver tissues using either Trizol

544 (Thermo Fisher Scientific; Waltham, MA, USA) or ToTALLY RNA kit (Thermo Fisher

545 Scientific; Waltham, MA, USA ) according to the manufacturers' instructions.

546 The obtained RNA was reverse-transcribed into cDNA using ReverTra Ace qPCR

547 RT Master Mix (TOYOBO; Osaka, Japan), and these were used as templates for real-time

548 PCR analyses. Real-time PCR was performed with KOD SYBR qPCR Mix (TOYOBO)

549 and primers (Supp Table 7) using the CFX Connect Real-Time PCR Detection System

550 (Bio-Rad; Hercules, CA, USA) according to the manufacturer's instructions. The

551 real-time PCR systems for $\mathrm{mmDeV}$ and $\operatorname{tgDeV}$ were validated using

552 pcDNA3-mmDeV(-) and pcDNA3-tgDeV(-) monomer, respectively, as controls.

553 End-point RT-PCR was also performed to confirm deltavirus infections. PCR was

554 performed with Phusion Hot Start II DNA Polymerase (Thermo Fisher Scientific) using

555 the above-described cDNAs and primers listed in Supp Table 7. The PCR products were

556 analyzed by agarose gel electrophoresis. The obtained PCR products were purified and 
sequenced by Sangar sequencing in FASMAC (Atsugi, Japan).

558

559

Determination of a full genome sequence of deltavirus in passerine birds

560 To determine the full genome sequence of detected deltaviruses, the cDNA obtained in

561 the section "Realtime and Endpoint RT-PCR detection of deltaviruses from animal

562 specimens" was amplified using illustra GenomiPhi V2 Kit (GE healthcare; Chicago, IL,

563 USA). The amplified DNA was then purified with innuPREP PCRpure Kit (Analytik

564 Jena: Jena, Germany). PCR was performed with Phusion Hot Start II DNA Polymerase

565 using the primers listed in Supp Table 7. The PCR products were analyzed using agarose

566 gel electrophoreses. When single bands were observed, the amplicon was purified with

567 innuPREP PCRpure Kit. When several bands were detected, bands of the expected sizes

568 were extracted and purified using Zymoclean Gel DNA Recovery Kit (Zymo Research).

569 The purified amplicons were sequenced in FASMAC (Atsugi, Japan).

$571 \quad$ Phylogenetic analysis

572 Deduced amino acid sequences of DAg proteins were used to infer the phylogenetic

573 relationship between deltaviruses. Multiple alignment was performed by MAFFT 7.427

574 using the E-INS-i algorithms (61), and ambiguously aligned regions were then removed

575 using trimAl 1.2rev59 with the --strict option (62). The phylogenetic relationship was

576 inferred by the maximum likelihood method using RAxML Next Generation v. 0.9.0 (63).

577 The LG+G model, which showed the lowest BIC by prottest3 3.4.2 (64), was used. The

578 reliability of the tree was assessed by 1,000 bootstrap resampling using the transfer

579 bootstrap expectation method (65). The alignment file is available in Supporting

580 materials. 
583 To identify co-infected viruses in deltavirus-positive SRAs, a three-step BLASTx search

584 was performed. First, BLASTx searches were performed against a custom database,

585 including RefSeq protein sequences from viruses using the assembled contigs (see the

586 subsection Detection of deltaviruses from publicly available transcriptome data) as

587 queries. The custom database was prepared as follows. Virus-derived protein sequences

588 in the RefSeq protein database (46) were downloaded on July 17, 2020, and were

589 clustered by cd-hit 4.8.1 (threshold $=0.9$ ). Then, sequences of more than 100 amino acid

590 residues were extracted using SeqKit 0.10.1 and these were used as a BLAST database.

591 The first BLAST hits were extracted, which were used for the second BLASTx analysis.

592 The second BLASTx analysis was performed against the NCBI RefSeq protein database.

593 The BLAST hits with the best hit to viral sequences were extracted and used for the final

594 BLASTx searches. The final BLASTx searches were performed against the NCBI nr

595 database. The BLAST hits with the best hit to viral sequences were extracted and

596 analyzed manually.

598 Cell culture

599 HepG2-NTCP cells were cultured with Dulbecco's modified Eagle's medium

600 (DMEM)/F-12 + GlutaMax (Thermo Fisher Scientific) supplemented with $10 \mathrm{mM}$

601 HEPES (Sigma Aldrich; St. Louis, MO, USA), 100 unit/ml penicillin (Meiji; Tokyo,

602 Japan), $100 \mathrm{mg} / \mathrm{ml}$ streptomycin (Meiji), 10\% FBS (Sigma Aldrich), $5 \mu \mathrm{g} / \mathrm{ml}$ insulin

603 (Wako; Tokyo, Japan) and 400 g/ml G418 (Nacalai tesque). Huh7 and WCH-17 cells

604 were maintained in DMEM (Wako) containing 10\% FBS (Sigma Aldrich), 100 unit/ml 
605 penicillin (Meiji), $100 \mathrm{mg} / \mathrm{ml}$ streptomycin (Meiji), $100 \mathrm{mM}$ nonessential amino acids

606 (Thermo Fisher Scientific), $1 \mathrm{mM}$ sodium pyruvate (Sigma Aldrich), and $10 \mathrm{mM}$ HEPES

607 (Sigma Aldrich).

608

609 Antibody production

610 The peptides corresponding to 65 to 78 aa (DSSSPRKRKRGEGG) of tgDeV DAg and

611174 to 187 aa (ESPYSRRGEGLDIR) of mmDeV DAg conjugated with cysteine at $\mathrm{N}$

612 terminus were synthesized. Each of the peptides was injected into mice, and antisera were

613 obtained from the mice at 42 days after the peptide injections. Each of the antisera was

614 affinity-purified using the corresponding peptide. The whole procedure was performed in

615 SCRUM (Tokyo, Japan).

617 Rescue of mmDeV and $\operatorname{tg} \mathrm{DeV}$

618 The DNA of negative-strand genomes of $\mathrm{mmDeV}$ and $\operatorname{tgDeV}$ was synthesized in

619 GenScript Japan (Tokyo, Japan). The synthesized DNAs were inserted into the KpnI

$620-\mathrm{XbaI}$ site of the pcDNA3 vector, designated as pcDNA3-mmDeV(-) monomer and

621 pcDNA-tgDeV (-) monomer. In addition, tandem sequences of mmDeV and tgDeV

622 genome were inserted into the pcDNA3 vector, which were named pcDNA3-mmDeV(-)

623 dimer and pcDNA-tgDeV (-) dimer, respectively. To rescue these viruses,

624 pcDNA3-mmDeV(-) dimer or pcDNA-tgDeV (-) dimer was transfected into Huh7 and

625 WCH-17 cells using Lipofectamine 3000 and Lipofectamine 2000 (Thermo Fisher

626 Scientific), respectively, according to the manufacturer's instructions. The transfected

627 cells were cultured for $48 \mathrm{~h}$ and were used for western blotting, IFA to verify DAg protein 628 expression. 
630 Western blotting

631 Cells were lysed with SDS sample buffer [100 mM Tris-HCl (pH 6.8) (Sigma Aldrich),

$6324 \%$ SDS (Nippon gene; Tokyo, Japan), $20 \%$ glycerol (Nacalai tesque), $10 \%$

633 2-mercaptoethanol (Wako)]. The cell lysates were subjected to SDS-PAGE and

634 transferred onto polyvinylidene difluoride membranes (Merck Millipore; Darmstadt,

635 Germany). After blocking the membranes with 5\% skim milk (Morinaga; Tokyo, Japan),

636 they were reacted with anti-tgDeV DAg, anti-mmDeV DAg, or anti-actin (Sigma

637 Aldrich) antibodies as primary antibodies, followed by reaction with horseradish

638 peroxidase (HRP)-conjugated secondary antibodies (Cell Signaling Technology;

639 Danvers, MA, USA).

640

641 Indirect immunofluorescence assay (IFA)

642 The cells were fixed in $4 \%$ paraformaldehyde (Wako) and then permeabilized using $0.3 \%$

643 Triton X-100 (MP Biomedicals; Santa Ana, CA, USA). After blocking the cells by

644 incubation in PBS containing 1\% bovine serum albumin (BSA) (KAC; Kyoto, Japan),

645 they were treated with the primary antibodies against $\mathrm{HDAg}, \operatorname{tgDeV} \mathrm{DAg}$, or mmDeV

646 DAg and then incubated with Alexa555-conjugated secondary antibody (Thermo Fisher

647 Scientific), together with DAPI (Nacalai tesque). To detect deltavirus-positive cells, the

648 fluorescence signal was observed using fluorescence microscopy, BZ-X710 (KEYENCE;

649 Osaka, Japan). High magnification examination of the subcellular localization was

650 performed using confocal microscopy, LSM900 (ZEISS; Oberkochen, Germany).

652 Deltavirus preparation and infection assay 
653 HDV was produced from the culture supernatants of Huh7 cells transfected with HDV

654 (pSVLD3) and HBs (pT7HB2.7) expressing plasmid, as described previously $(32,66)$.

$655 \operatorname{tgDeV}$ and $\mathrm{mmDeV}$ were also subjected to the same assay. The supernatants of

656 transfected cells were collected at 6,9 , and 12 days post-transfection, and they were then

657 filtrated and concentrated using 0.45- $\mu \mathrm{m}$ filters and Amicon Ultra (Merck Millipore),

658 according to the manufacturer's instructions. The concentrated supernatants were

659 inoculated into HepG2-NTCP cells with 5\% PEG8000 (Sigma Aldrich) for $24 \mathrm{~h}$ followed

660 by washing to remove free viruses. The inoculated cells were cultured for 6 days and used

661 for the downstream analyses.

662

663 Acknowledgments

664 HDV and HBs expression plasmids were kindly provided by Dr. John Taylor (the Fox

665 Chase Cancer) and Dr. Camille Sureau (Institute National de la Transfusion Sanguine).

666 We thank Dr. Keiko Takemoto (Kyoto University) for her kind help with the setting of

667 computer resources. The super-computing resources were provided by Human Genome

668 Center, the Institute of Medical Science, the University of Tokyo, and the NIG

669 supercomputer at ROIS National Institute of Genetics. All the silhouette images except

670 for woodchuck were downloaded from silhouetteAC (http://www.silhouette-ac.com/).

671 This study was supported by the Hakubi project at Kyoto University (MH);

672 Grant-in-Aid for Scientific Research on Innovative Areas from the Ministry of Education,

673 Culture, Science, Sports, and Technology (MEXT) of Japan, Grant Numbers

674 JP19H04839 (SI), JP16H06429 (KT), JP16K21723 (KT), JP16H0643 (KT),

675 JP17H05821 (MH), and JP19H04833 (MH); the Japan Society for the Promotion of

676 Science KAKENHI, Grant Numbers JP19K16672 (MI), JP20J00868 (MI), and 
677 JP20H03499 (KW); AMED Grant Numbers JP20jm0210068j0002 (KW) and

678 JP20fk0310114j0004 (KW).

679

680 Author contributions

681 MH conceived the study. MI, KW, and MH designed the study. MI conducted cell culture

682 experiments. MH, JK, SK performed in silico analyses. YS, YTL, HLW, KW, and MH

683 prepared and analyzed animal specimens. All the authors analyzed and discussed the data.

684 MI and MH wrote the manuscript.

685 


\section{Figure legends}

687 Figure 1. Genome organization of novel deltaviruses.

688 Genomes of (a) tgDeV, mmDeV, and ovDeV (complete genomes) and (b) scDeV and

689 egDeV (partial genomes). Annotations (ORF, poly-A signal, and ribozymes) are shown

690 by colored arrow pentagons. The numbers indicate nucleotide positions. (c)

691 Self-complementarities of novel deltaviruses. The predicted RNA structures were

692 visualized using the Mfold web server (58). Red, blue, and green arcs indicate G-C, A-U, 693 and G-U pairs, respectively.

Figure 2. Amino acid sequence characterization of putative delta antigens of novel

\section{6 deltaviruses.}

697 (a) Alignment and functional features of the putative S-HDAg and DAgs of

698 representative HDVs and novel deltaviruses. (Putative) functional domains are shown by

699 colored boxes. Me: arginine methylation site, Ac: lysine acetylation site, P: Serine

700 phosphorylation site. (b) ovDeV mRNA (upper panel) and a possible A-to-I RNA-editing

701 site (lower panel). Consensus ovDeV-DAg mRNA sequence and mapped read sequence

702 with potential RNA-edited nucleotides (blue boxes). Pink boxes indicate the ORF of

703 ovDeV DAg. (c) Deduced amino acid sequences of ovDeV-DAg proteins translated from

704 the viral mRNA with or without RNA-editing. The blue letter shows the possible

705 RNA-editing site.

706

707 Figure 3. Mapping coverages of original short reads of each contig.

708 Mapped read graphs of (a) tgDeV, (b) $\mathrm{mmDeV}$, and (c) ovDeV. Lines, arrow pentagons,

709 and arrowheads indicate viral genomes, ribozymes, and poly(A) signals, respectively. 
710 The numbers above the graphs show nucleotide positions. The light pink box indicates a

711 low read depth region in the putative transcript of $\operatorname{tgDeV}$.

712

713 Figure 4. Interfamily transmission of deltaviruses among passerine birds.

714 (a-c) RT-PCR detection of a deltavirus from Lonchura striata. (a) Plasmid used for the

715 establishment of real-time PCR detection system for tgDeV and (b) the tgDeV circular

716 genome. The blue arrows indicate the primers used for endo-point RT-PCR detection. (c)

717 Endo-point RT-PCR for detection of the circular deltavirus genome. M, 100-bp ladder

718 marker. (d) Pairwise nucleotide identities between deltaviruses detected in passerine

719 birds. (e) Phylogenetic tree of passerine birds positive for deltaviruses. Phylogenetic tree

720 of birds and deltavirus infections are indicated. MYA: million years ago.

721

722 Figure 5. Phylogenetic analysis of deltaviruses.

723 (a) Heat map of pairwise amino acid sequence identities between deltaviruses. (b) The

724 phylogenetic tree was inferred by the maximum likelihood method using an amino acid

725 sequence alignment of representative deltaviruses. Known phenotypes (RNA-editing and

726 expression of the large isoform of DAg protein) and helper virus(es) of each virus are

727 shown on the right. Note that the $\mathrm{SDeV}$ phenotypes are shown in gray letters, because

728 there is insufficient information, evidence, or both for the RNA-editing and L-DAg

729 expression. The deltaviruses identified in this study are indicated by the blue circles.

730 Bootstrap values $>70$ are shown. SDeV: snake deltavirus, RDeV: rodent deltavirus.

732 Figure 6. Detection of tgDeV DAg and mmDeV DAg in cells ectopically expressing

733 the tgDeV or mmDeV dimer genome. 
734 (a and b) Western blotting analysis of Huh7 or WCH-17 cells transfected with a tgDeV or

$735 \mathrm{mmDeV}$ dimer-sequence expression plasmid. The numbers on the left side of panels

736 indicate the size marker of protein $(\mathrm{kDa}) .(\mathbf{c}-\mathbf{f})$ Indirect immunofluorescence analysis of

737 the expression of $\operatorname{tgDeV}$ or mmDeV DAg protein. The cells were observed using

738 fluorescent microscopy (c and d) or a confocal microscopy (e and f). Blue; DAPI, Red;

$739 \operatorname{tgDeV}$ or mmDeV DAg. Scale bars $=50 \mu \mathrm{m}(\mathrm{c}$ and $\mathrm{d})$ and $5 \mu \mathrm{m}(\mathrm{e}$ and $\mathrm{f})$.

741 Figure 7. No infectious particle of $\operatorname{tgDeV}$ and $\mathrm{mmDeV}$ was produced by 742 supplementation of $\mathrm{HBV}$ envelop proteins.

743 (a) Quantification of deltavirus RNAs in culture supernatants. HDV, tgDeV, or mmDeV

744 expression plasmid was transfected with or without plasmid expressing HBV envelope

745 proteins into Huh7 cells. Viral RNA levels in supernatants were quantified using

746 quantitative RT-PCR $(n=3)$. (b and c) HepG2-NTCP cells were incubated with the

747 culture supernatants of the transfectants for $24 \mathrm{~h}$ in the presence or absence of $500 \mathrm{nM}$

748 Myrcludex B (MyrB), an inhibitor of HBV envelope-dependent viral entry. The cells

749 were cultured for an additional 6 days, and viral RNA levels and protein exprssion were

750 analyzed using quantitative RT-PCR $(n=3)(\mathbf{b})$ and IFA $(\mathbf{c})$, respectively. The numbers in

751 (c) correspond to those of (b). Blue, DAPI; Red, HDV; $\operatorname{tgDeV}$, or mmDeV DAg. Scale

752 bar $=50 \mu \mathrm{m}$. 


\section{Reference}

755 1. Magnius L, Taylor J, Mason WS, Sureau C, Deny P, Norder H, Ictv Report C.

756 2018. ICTV Virus Taxonomy Profile: Deltavirus. J Gen Virol 99:1565-1566.

757 2. Wong SK, Lazinski DW. 2002. Replicating hepatitis delta virus RNA is edited in

758 the nucleus by the small form of ADAR1. Proc Natl Acad Sci U S A

759 99:15118-23.

760 3. Bergmann KF, Gerin JL. 1986. Antigens of hepatitis delta virus in the liver and

761 serum of humans and animals. J Infect Dis 154:702-6.

762 4. Bonino F, Heermann KH, Rizzetto M, Gerlich WH. 1986. Hepatitis delta virus:

763 protein composition of delta antigen and its hepatitis B virus-derived envelope. $\mathrm{J}$

$764 \quad$ Virol 58:945-50.

765 5. Taylor JM. 2020. Infection by Hepatitis Delta Virus. Viruses 12.

766 6. O'Malley B, Lazinski DW. 2005. Roles of carboxyl-terminal and farnesylated

767 residues in the functions of the large hepatitis delta antigen. J Virol 79:1142-53.

$7687 . \quad$ Kuo MY, Sharmeen L, Dinter-Gottlieb G, Taylor J. 1988. Characterization of

769 self-cleaving RNA sequences on the genome and antigenome of human hepatitis

770 delta virus. J Virol 62:4439-44.

771 8. Sharmeen L, Kuo MY, Dinter-Gottlieb G, Taylor J. 1988. Antigenomic RNA of

772 human hepatitis delta virus can undergo self-cleavage. J Virol 62:2674-9.

773 9. Chen PJ, Kalpana G, Goldberg J, Mason W, Werner B, Gerin J, Taylor J. 1986.

774 Structure and replication of the genome of the hepatitis delta virus. Proc Natl

$775 \quad$ Acad Sci U S A 83:8774-8.

776 10. Kos A, Dijkema R, Arnberg AC, van der Meide PH, Schellekens H. 1986. The

777 hepatitis delta (delta) virus possesses a circular RNA. Nature 323:558-60.

778 11. Wang KS, Choo QL, Weiner AJ, Ou JH, Najarian RC, Thayer RM, Mullenbach

779 GT, Denniston KJ, Gerin JL, Houghton M. 1986. Structure, sequence and

780 expression of the hepatitis delta (delta) viral genome. Nature 323:508-14.

781 12. Rizzetto M. 2015. Hepatitis D Virus: Introduction and Epidemiology. Cold

$782 \quad$ Spring Harb Perspect Med 5:a021576.

783 13. Littlejohn M, Locarnini S, Yuen L. 2016. Origins and Evolution of Hepatitis B

784 Virus and Hepatitis D Virus. Cold Spring Harb Perspect Med 6:a021360.

785 14. Rizzetto M. 2016. The adventure of delta. Liver Int 36 Suppl 1:135-40.

786 15. Wille M, Netter HJ, Littlejohn M, Yuen L, Shi M, Eden JS, Klaassen M, Holmes 
EC, Hurt AC. 2018. A Divergent Hepatitis D-Like Agent in Birds. Viruses 10.

16. Chang WS, Pettersson JH, Le Lay C, Shi M, Lo N, Wille M, Eden JS, Holmes EC. 2019. Novel hepatitis D-like agents in vertebrates and invertebrates. Virus Evol 5:vez021.

17. Hetzel U, Szirovicza L, Smura T, Prahauser B, Vapalahti O, Kipar A, Hepojoki J. 2019. Identification of a Novel Deltavirus in Boa Constrictors. mBio 10.

18. Paraskevopoulou S, Pirzer F, Goldmann N, Schmid J, Corman VM, Gottula LT, Schroeder S, Rasche A, Muth D, Drexler JF, Heni AC, Eibner GJ, Page RA, Jones TC, Muller MA, Sommer S, Glebe D, Drosten C. 2020. Mammalian deltavirus without hepadnavirus coinfection in the neotropical rodent Proechimys semispinosus. Proc Natl Acad Sci U S A doi:10.1073/pnas.2006750117.

19. Szirovicza L, Hetzel U, Kipar A, Martinez-Sobrido L, Vapalahti O, Hepojoki J. 2020. Snake Deltavirus Utilizes Envelope Proteins of Different Viruses To Generate Infectious Particles. mBio 11.

20. Hedges SB, Dudley J, Kumar S. 2006. TimeTree: a public knowledge-base of divergence times among organisms. Bioinformatics 22:2971-2.

21. Chao YC, Tang HS, Hsu CT. 1994. Evolution rate of hepatitis delta virus RNA isolated in Taiwan. J Med Virol 43:397-403.

22. Krushkal J, Li WH. 1995. Substitution rates in hepatitis delta virus. J Mol Evol 41:721-6.

23. Alvarado-Mora MV, Romano CM, Gomes-Gouvea MS, Gutierrez MF, Carrilho FJ, Pinho JR. 2011. Dynamics of hepatitis D (delta) virus genotype 3 in the Amazon region of South America. Infect Genet Evol 11:1462-8.

24. Fletcher SP, Chin DJ, Ji Y, Iniguez AL, Taillon B, Swinney DC, Ravindran P, Cheng DT, Bitter H, Lopatin U, Ma H, Klumpp K, Menne S. 2012.

Transcriptomic analysis of the woodchuck model of chronic hepatitis B. Hepatology 56:820-30.

25. Fletcher SP, Chin DJ, Gruenbaum L, Bitter H, Rasmussen E, Ravindran P, Swinney DC, Birzele F, Schmucki R, Lorenz SH, Kopetzki E, Carter J, Triyatni M, Thampi LM, Yang J, AlDeghaither D, Murreddu MG, Cote P, Menne S. 2015. Intrahepatic Transcriptional Signature Associated with Response to Interferon-alpha Treatment in the Woodchuck Model of Chronic Hepatitis B. 
$820 \quad$ PLoS Pathog 11:e1005103.

821 26. Ryu WS, Bayer M, Taylor J. 1992. Assembly of hepatitis delta virus particles. J

$822 \quad$ Virol 66:2310-5.

823 27. Gudima S, He Y, Chai N, Bruss V, Urban S, Mason W, Taylor J. 2008. Primary

824 human hepatocytes are susceptible to infection by hepatitis delta virus

825 assembled with envelope proteins of woodchuck hepatitis virus. J Virol

$826 \quad 82: 7276-83$.

827 28. Zuccola HJ, Rozzelle JE, Lemon SM, Erickson BW, Hogle JM. 1998. Structural

828 basis of the oligomerization of hepatitis delta antigen. Structure 6:821-830.

829 29. Noe L, Kucherov G. 2005. YASS: enhancing the sensitivity of DNA similarity

830 search. Nucleic Acids Res 33:W540-3.

831 30. Yan H, Zhong G, Xu G, He W, Jing Z, Gao Z, Huang Y, Qi Y, Peng B, Wang H,

832 Fu L, Song M, Chen P, Gao W, Ren B, Sun Y, Cai T, Feng X, Sui J, Li W. 2012.

833 Sodium taurocholate cotransporting polypeptide is a functional receptor for

834 human hepatitis B and D virus. Elife 1:e00049.

835 31. Iwamoto M, Watashi K, Tsukuda S, Aly HH, Fukasawa M, Fujimoto A, Suzuki

836 R, Aizaki H, Ito T, Koiwai O, Kusuhara H, Wakita T. 2014. Evaluation and identification of hepatitis B virus entry inhibitors using HepG2 cells overexpressing a membrane transporter NTCP. Biochem Biophys Res Commun 443:808-13.

32. Sureau C, Guerra B, Lee H. 1994. The middle hepatitis B virus envelope protein is not necessary for infectivity of hepatitis delta virus. J Virol 68:4063-6.

35. Edgar RC, Taylor J, Altman T, Barbera P, Meleshko D, Lin V, Lohr D,

33. Gripon P, Cannie I, Urban S. 2005. Efficient inhibition of hepatitis B virus infection by acylated peptides derived from the large viral surface protein. J Virol 79:1613-22.

34. Bergner LM, Orton RJ, Broos A, Tello C, Becker DJ, Carrera JE, Patel AH, Biek R, Streicker DG. 2020. Diversification of mammalian deltaviruses by host shifting. bioRxiv doi:10.1101/2020.06.17.156745.

852 36. Anonymous. !!! INVALID CITATION !!! $(34,35)$. 
37. Chang FL, Chen PJ, Tu SJ, Wang CJ, Chen DS. 1991. The large form of hepatitis delta antigen is crucial for assembly of hepatitis delta virus. Proc Natl Acad Sci U S A 88:8490-4.

39. Kircher M, Sawyer S, Meyer M. 2012. Double indexing overcomes inaccuracies

40. Nelson MC, Morrison HG, Benjamino J, Grim SL, Graf J. 2014. Analysis,

41. Renaud G, Stenzel U, Maricic T, Wiebe V, Kelso J. 2015. deML: robust

48. Kim D, Paggi JM, Park C, Bennett C, Salzberg SL. 2019. Graph-based genome optimization and verification of Illumina-generated 16S rRNA gene amplicon surveys. PLoS One 9:e94249.

42. D'Amore R, Ijaz UZ, Schirmer M, Kenny JG, Gregory R, Darby AC, Shakya M, ing Bioinformatics 31:770-2. Podar M, Quince C, Hall N. 2016. A comprehensive benchmarking study of protocols and sequencing platforms for $16 \mathrm{~S}$ rRNA community profiling. BMC Genomics 17:55.

43. Wright ES, Vetsigian KH. 2016. Quality filtering of Illumina index reads mitigates sample cross-talk. BMC Genomics 17:876.

44. Fuxjager MJ, Lee JH, Chan TM, Bahn JH, Chew JG, Xiao X, Schlinger BA. 2016. Research Resource: Hormones, Genes, and Athleticism: Effect of Androgens on the Avian Muscular Transcriptome. Mol Endocrinol 30:254-71.

45. Duffy DL, Bentley GE, Drazen DL, Ball GF. 2000. Effects of testosterone on cell-mediated and humoral immunity in non-breeding adult European starlings. Behavioral Ecology 11:654-662.

46. Coordinators NR. 2018. Database resources of the National Center for Biotechnology Information. Nucleic Acids Res 46:D8-D13.

47. Chen S, Zhou Y, Chen Y, Gu J. 2018. fastp: an ultra-fast all-in-one FASTQ preprocessor. Bioinformatics 34:i884-i890. alignment and genotyping with HISAT2 and HISAT-genotype. Nat Biotechnol 37:907-915. 
886 49. Li H, Handsaker B, Wysoker A, Fennell T, Ruan J, Homer N, Marth G, Abecasis Alignment/Map format and SAMtools. Bioinformatics 25:2078-9.

50. Bankevich A, Nurk S, Antipov D, Gurevich AA, Dvorkin M, Kulikov AS, Lesin

57. Byun Y, Han K. 2009. PseudoViewer3: generating planar drawings of

58. Zuker M. 2003. Mfold web server for nucleic acid folding and hybridization

59. Ludwiczak J, Winski A, Szczepaniak K, Alva V, Dunin-Horkawicz S. 2019. VM, Nikolenko SI, Pham S, Prjibelski AD, Pyshkin AV, Sirotkin AV, Vyahhi N, Tesler G, Alekseyev MA, Pevzner PA. 2012. SPAdes: a new genome assembly algorithm and its applications to single-cell sequencing. J Comput Biol 19:455-77.

51. Nurk S, Meleshko D, Korobeynikov A, Pevzner PA. 2017. metaSPAdes: a new

52. Li W, Godzik A. 2006. Cd-hit: a fast program for clustering and comparing large sets of protein or nucleotide sequences. Bioinformatics 22:1658-9.

53. Fu L, Niu B, Zhu Z, Wu S, Li W. 2012. CD-HIT: accelerated for clustering the next-generation sequencing data. Bioinformatics 28:3150-2.

54. Shen W, Le S, Li Y, Hu F. 2016. SeqKit: A Cross-Platform and Ultrafast Toolkit for FASTA/Q File Manipulation. PLoS One 11:e0163962.

55. Camacho C, Coulouris G, Avagyan V, Ma N, Papadopoulos J, Bealer K, Madden TL. 2009. BLAST+: architecture and applications. BMC Bioinformatics 10:421. DeepCoil-a fast and accurate prediction of coiled-coil domains in protein sequences. Bioinformatics 35:2790-2795.

60. Barnett DW, Garrison EK, Quinlan AR, Stromberg MP, Marth GT. 2011. BamTools: a C++ API and toolkit for analyzing and managing BAM files. Bioinformatics 27:1691-2.

1. Katoh K, Standley DM. 2013. MAFFT multiple sequence alignment software version 7: improvements in performance and usability. Mol Biol Evol 30:772-80. 
919 62. Capella-Gutierrez S, Silla-Martinez JM, Gabaldon T. 2009. trimAl: a tool for

920 automated alignment trimming in large-scale phylogenetic analyses.

921 Bioinformatics 25:1972-3.

922 63. Kozlov AM, Darriba D, Flouri T, Morel B, Stamatakis A. 2019. RAxML-NG: a

923 fast, scalable and user-friendly tool for maximum likelihood phylogenetic

924 inference. Bioinformatics 35:4453-4455.

925 64. Darriba D, Taboada GL, Doallo R, Posada D. 2011. ProtTest 3: fast selection of

926 best-fit models of protein evolution. Bioinformatics 27:1164-5.

927 65. Lemoine F, Domelevo Entfellner JB, Wilkinson E, Correia D, Davila Felipe M,

928 De Oliveira T, Gascuel O. 2018. Renewing Felsenstein's phylogenetic bootstrap

929 in the era of big data. Nature 556:452-456.

930 66. Kuo MY, Chao M, Taylor J. 1989. Initiation of replication of the human hepatitis

931 delta virus genome from cloned DNA: role of delta antigen. J Virol 63:1945-50.

932 
Table 1. Summary of novel deltaviruses

\begin{tabular}{|c|c|c|c|c|c|c|c|c|c|c|}
\hline \multirow{2}{*}{ Virus name } & & \multirow{2}{*}{ Host species } & \multirow{2}{*}{ Tissue } & \multirow{2}{*}{$\begin{array}{c}\text { SRA } \\
\text { accession }\end{array}$} & \multirow{2}{*}{$\begin{array}{c}\text { DDBJ } \\
\text { accession }\end{array}$} & \multirow{2}{*}{$\begin{array}{c}\text { Contig } \\
\text { length (nt) }\end{array}$} & \multirow{2}{*}{$\begin{array}{c}\text { GC content } \\
(\%)\end{array}$} & \multicolumn{3}{|c|}{ BLASTx best hit } \\
\hline & & & & & & & & Virus name & Accession & Identity (\%) \\
\hline Taeniopygia guttata $\mathrm{DeV}$ & $\operatorname{tgDeV}$ & Taeniopygia guttata & $\begin{array}{l}\text { Scapulohumeralis } \\
\text { caudalis }\end{array}$ & SRR2545946 & BR001665 & 1706 & 56.6 & Rodent deltavirus & QJD13558 & 63.3 \\
\hline Marmota monax DeV & $\mathrm{mmDeV}$ & Marmota monax & Liver & SRR2136906 & BR001661 & 1712 & 53.4 & Hepatitis delta virus & AIR77039 & 60.0 \\
\hline Odocoileus virginianus DeV & ovDeV & Odocoileus virginianus & Pedicle & SRR4256033 & BR001662 & 1690 & 56.4 & Hepatitis delta virus & AHB60712 & 66.7 \\
\hline Erythrura gouldiae DeV & egDeV & Erythrura gouldiae & Skin & SRR7504989 & BR001660 & 596 & $59.4^{\text {a) }}$ & Rodent deltavirus & QJD13555 & 63.5 \\
\hline Serinus canaria-associated $\mathrm{DeV}$ & $\mathrm{scDeV}$ & Serinus canaria & Skin & SRR2915371 & BR001664 & 761 & $54.4^{\mathrm{a})}$ & Hepatitis delta virus & AIR77012 & 36.0 \\
\hline Pardaliparus venustulus DeV & pvDeV & Pardaliparus venustulus & $\begin{array}{l}\text { Lung, Kidney, } \\
\text { Cardiac muscle, } \\
\text { Flight muscle, } \\
\text { Liver }\end{array}$ & $\begin{array}{l}\text { SRR7244693 } \\
\text { SRR7244695 } \\
\text { SRR7244696 } \\
\text { SRR7244697 } \\
\text { SRR7244698 }\end{array}$ & BR001663 & 1708 & 55.8 & Rodent deltavirus & QJD13562 & 62.4 \\
\hline Lonchura striata $\mathrm{DeV}$ & $1 \mathrm{sDeV}$ & $\begin{array}{l}\text { Lonchura striata var. } \\
\text { domestica }\end{array}$ & Blood & - & LC575944 & 1708 & 56.2 & Rodent deltavirus & QJD13555 & 62.9 \\
\hline
\end{tabular}

a) GC content of the partial genome sequences. 
bioRxiv preprint doi: https://doi.org/10.1101/2020.08.30.274571; this version posted October 18,2020 . The copyright holder for this preprint (which was not certified by peer review) is the author/funder, who has granted bioRxiv a license to display the preprint in perpetuity. It is made available under aCC-BY-NC-ND 4.0 International license.

\section{Table 2. Detection of deltavirus-derived reads in RNA-seq data.}

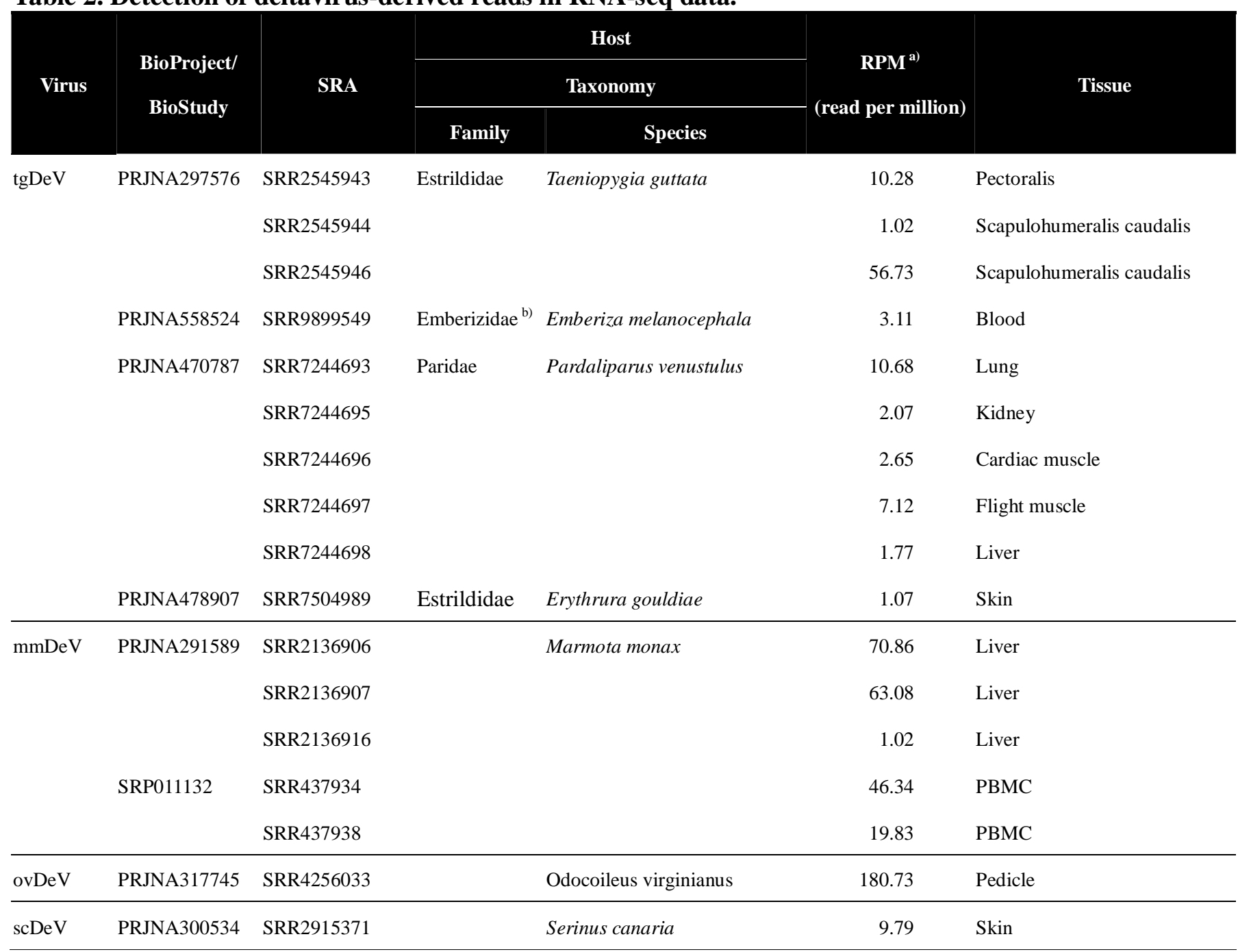

The full version of the table is available as Supplementary Table 3.

a) This table only shows the samples with RPM $>1$.

b) Emberizidae is regarded as the subfamily Emberizinae of the family Fringillidae in TimeTree. 
bioRxiv preprint doi: https://doi.org/10.1101/2020.08.30.274571; this version posted October 18, 2020. The copyright holder for this preprint (which was not certified by peer review) is the author/funder, who has granted bioRxiv a license to display the preprint in perpetuity. It is made available under aCC-BY-NC-ND 4.0 International license.

\section{Table 3. Coexisting viruses in deltavirus-positive SRAs}

\begin{tabular}{|c|c|c|c|c|c|}
\hline SRA accession & \multicolumn{2}{|c|}{ Host } & Virus name & Envelope & $\begin{array}{l}\text { Deltavirus } \\
\text { infection }\end{array}$ \\
\hline SRR2545944 & Taeniopygia guttata & Zebra finch & Serinus canaria polyomavirus & - & $\operatorname{tg} \mathrm{DeV}$ \\
\hline SRR5001849 & Taeniopygia guttata & Zebra finch & Serinus canaria polyomavirus & - & $\operatorname{tg} \mathrm{DeV}$ \\
\hline SRR5001850 & Taeniopygia guttata & Zebra finch & Serinus canaria polyomavirus & - & $\operatorname{tgDeV}$ \\
\hline SRR5001851 & Taeniopygia guttata & Zebra finch & Serinus canaria polyomavirus & - & $\operatorname{tg} \mathrm{DeV}$ \\
\hline SRR2915371 & Serinus canaria & Common canary & Canary bornavirus 3 & + & $\mathrm{scDeV}$ \\
\hline & & & Canary circovirus & - & \\
\hline SRR7504989 & Erythrura gouldiae & Gouldian finch & Erythrura gouldiae polyomavirus 1 & - & egDeV \\
\hline
\end{tabular}



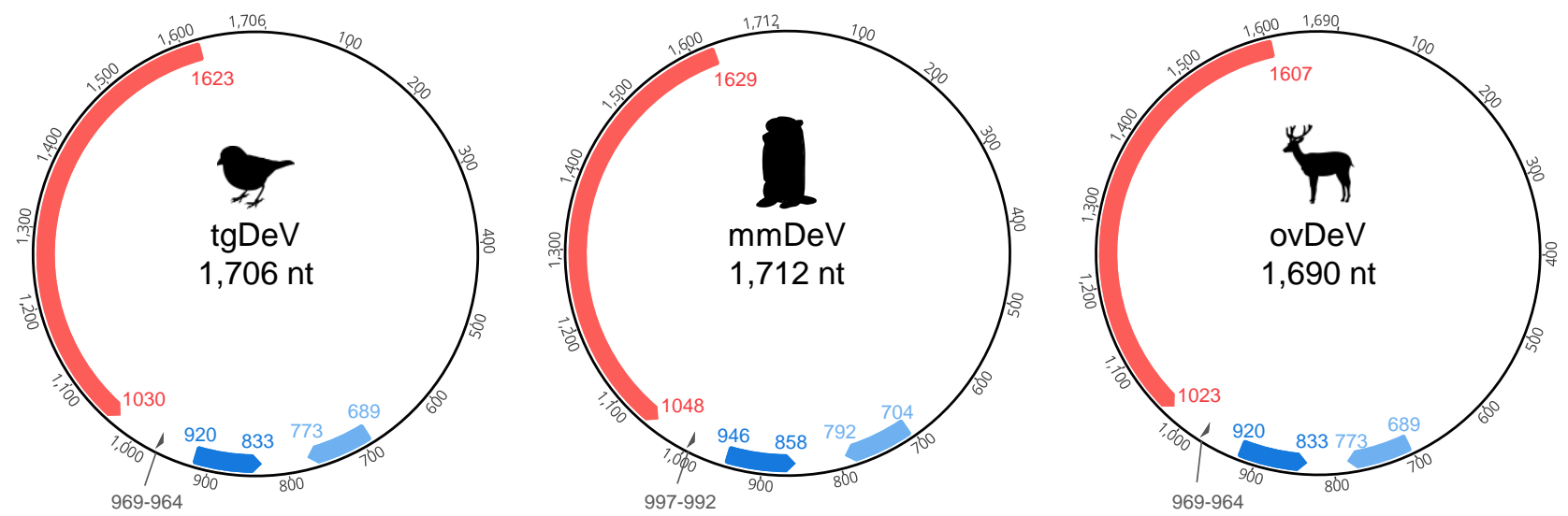

b
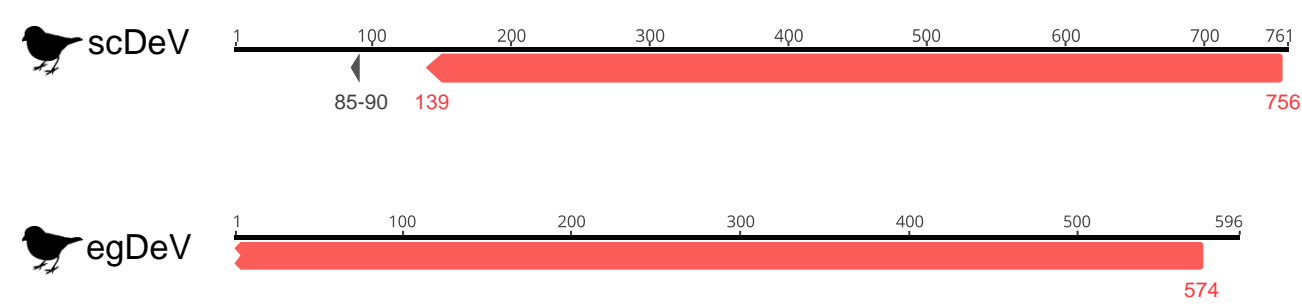

C
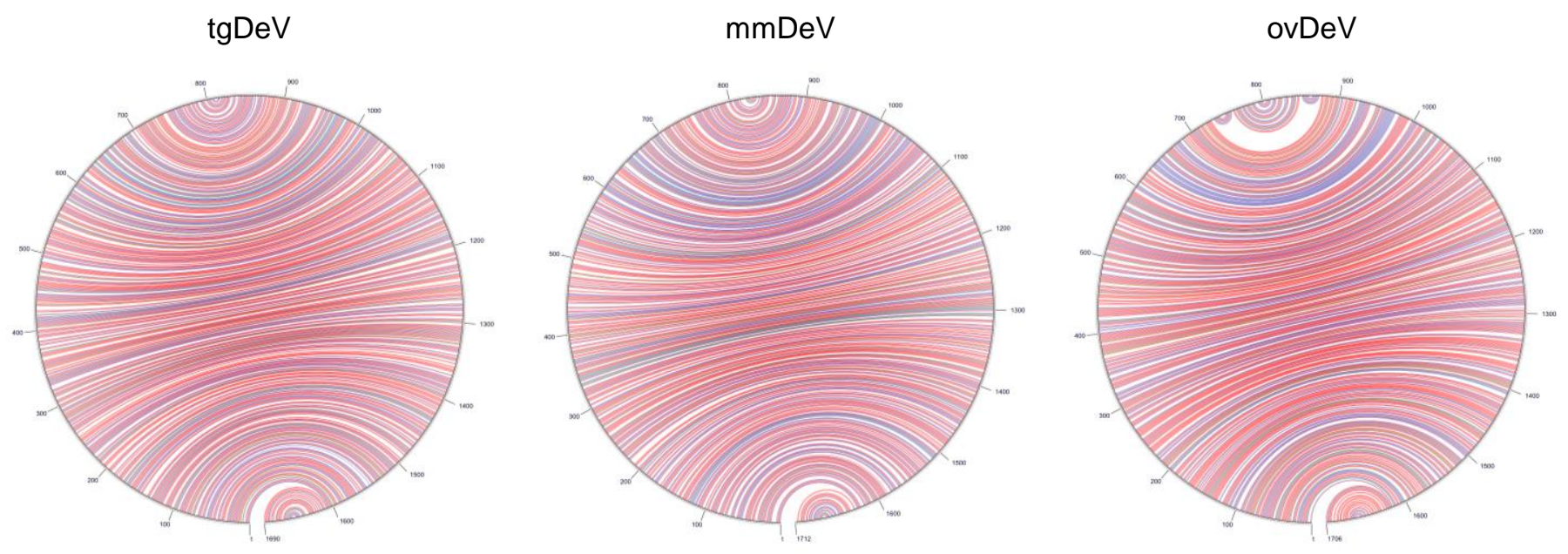
a

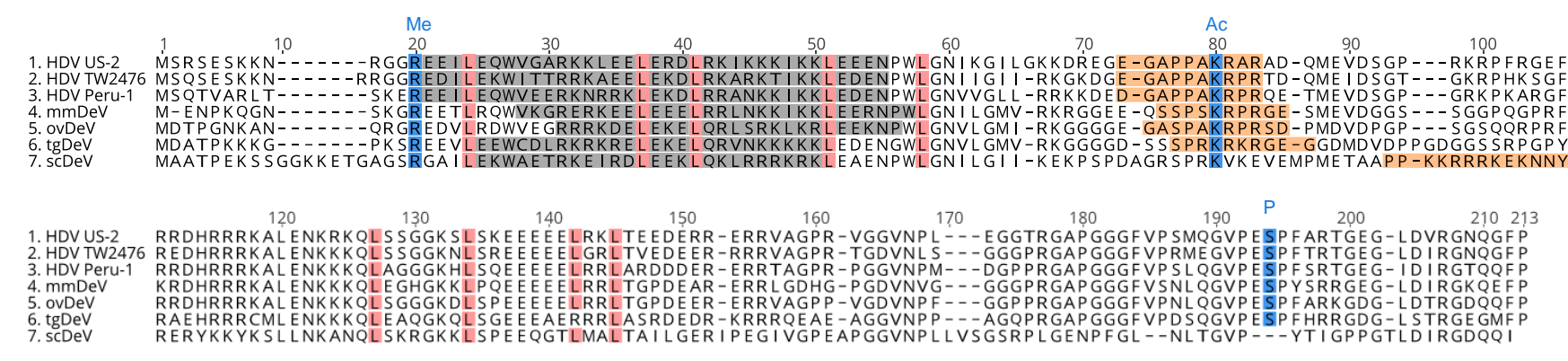

Conserved post-translational modification sites $\quad$ (Predicted) NLS $\quad$ Me Methylation site P Phosphorylation site

Conserved leucine residues $\quad$ (Predicted) coiled-coil domain Ac Acetylation site

b

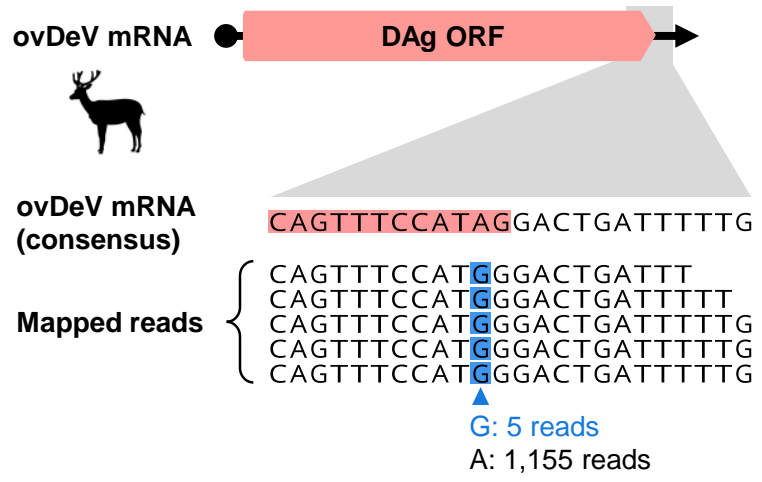

A: 1,155 reads
C

A-to-I editing \begin{tabular}{cccccccc}
...CAG UUU & CCA & UGG & GAC UGA UUU UUG... \\
$Q$ & $F$ & $P$ & $W$ & $D$ & $*$ \\
\hline
\end{tabular} 
Fig. 3

a

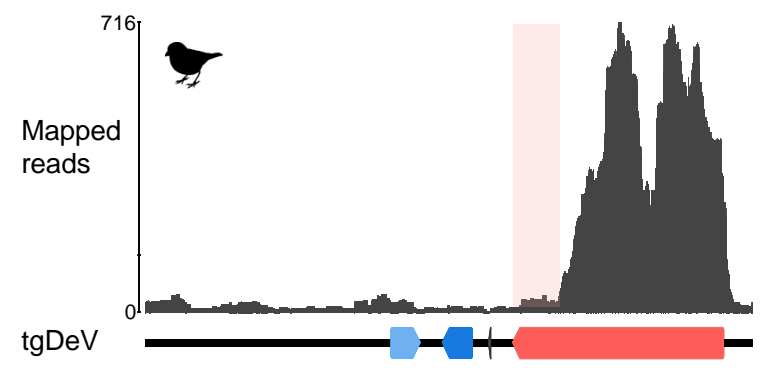

C

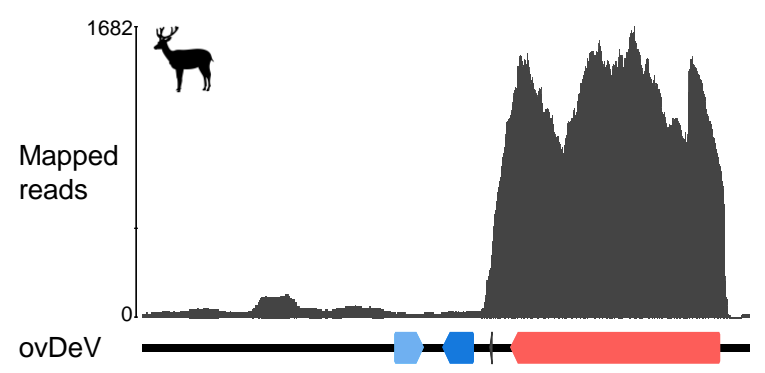

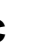

b

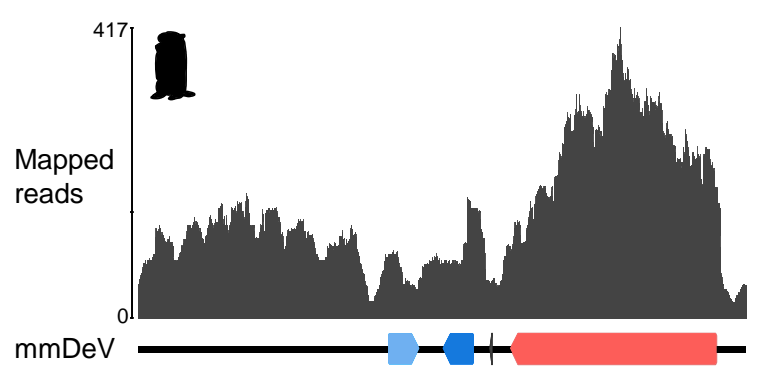

d

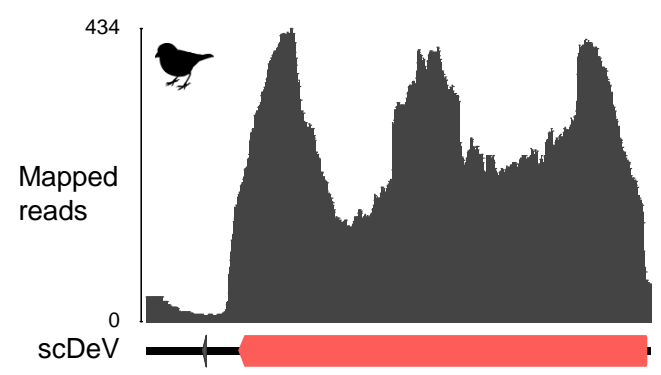
Delta antigen ORF
Putative genomic ribozyme
Poly $(A)$ signal
Putative antigenomic ribozyme
Poly $(A)$ signal

(which was not certified by peer review) is the author/funder, who has granted bioRxiv a license to display the preprint in perpetuity. It is $m$ 
a

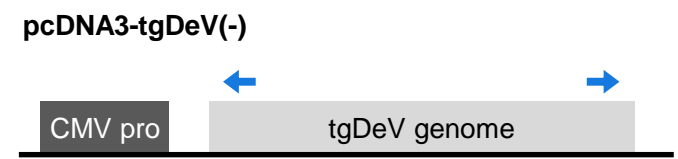

d

\begin{tabular}{cccc}
\multicolumn{4}{c}{ Nucleotide identity (\%) } \\
\hline & pvDeV & $\operatorname{tgDeV}$ & IsDeV \\
\hline pvDeV & & 98.2 & 98.4 \\
tgDeV & 98.2 & & 98.2 \\
IsDeV & 98.4 & 98.2 & \\
\hline
\end{tabular}

b

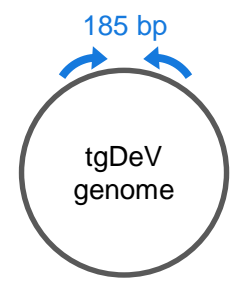

e

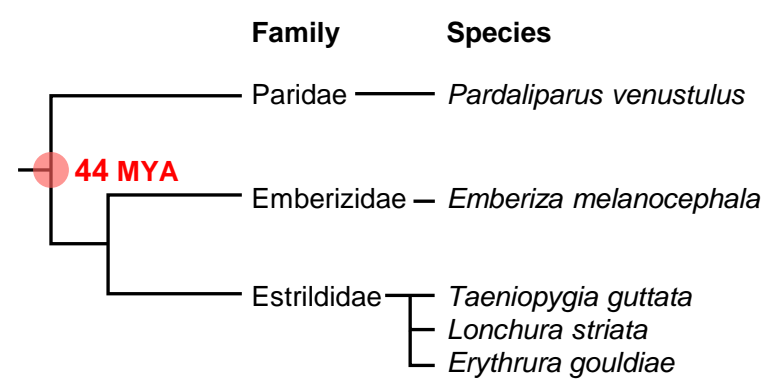

Deltavirus

reads

+ pvDeV

$+\quad \operatorname{tgDeV}$

+ IsDeV

$+\quad$ egDeV 
a

\begin{tabular}{|c|c|c|c|c|c|c|c|c|c|c|c|c|c|c|c|c|c|c|c|}
\hline & 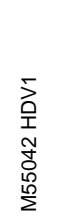 & 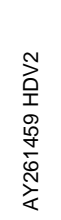 & 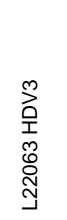 & 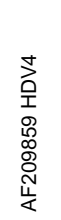 & 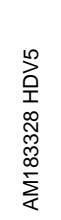 & 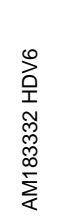 & 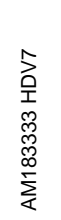 & 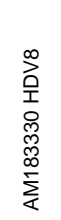 & $\begin{array}{l}\text { ऐे } \\
\text { Oे }\end{array}$ & $\begin{array}{l}\vec{\nabla} \\
\stackrel{\text { }}{E} \\
\text { है }\end{array}$ & 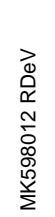 & $\begin{array}{l}\text { ఖे } \\
\text { Oे }\end{array}$ & 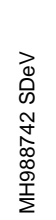 & 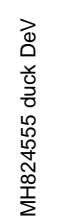 & 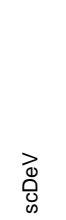 & 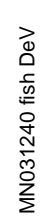 & 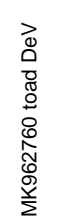 & 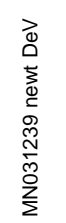 & 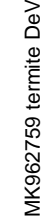 \\
\hline M55042 HDV1 & & 75.0 & 67.9 & 74.0 & 73.0 & 70.9 & 72.6 & 74.0 & 63.3 & 57.7 & 55.1 & 51.3 & 48.5 & 40.2 & 35.0 & 22.4 & 21.5 & 23.6 & 21.3 \\
\hline AY261459 HDV2 & 75.0 & & 6.2 & 74.9 & 76.5 & 73.3 & 72.4 & 76.9 & 62.6 & 56.4 & 54.8 & 51.5 & 46.2 & 35.6 & 37.1 & 19.0 & 24.6 & 25.6 & 20.4 \\
\hline L22063 HDV3 & 67.9 & 66.2 & & 64.9 & 63.1 & 62.1 & 64.2 & 63.1 & 62.4 & 60.3 & 55.6 & 54.3 & 49.7 & 36.7 & 35.1 & 19.0 & 20.6 & 20.0 & 20.4 \\
\hline AF209859 HDV4 & 74.0 & 74.9 & 64.9 & & 72.4 & 73.3 & 74.0 & 76.9 & 67.0 & 60.8 & 56.1 & 52.8 & 47.2 & 39.4 & 33.2 & 19.0 & 23.6 & 23.3 & 20.4 \\
\hline AM183328 HDV5 & 73.0 & 76.5 & 63.1 & 72.4 & & 74.4 & 74.5 & 79.5 & 62.6 & 53.4 & 52.3 & 50.0 & 45.3 & 36.8 & 38.1 & 20.0 & 23.7 & 24.7 & 22.4 \\
\hline AM183332 HDV6 & 70.9 & 73.3 & 62.1 & 73.3 & 74.4 & & 71.9 & 77.4 & 66.7 & 54.4 & 54.8 & 52.0 & 48.7 & 37.2 & 37.1 & 20.6 & 22.6 & 23.3 & 21.5 \\
\hline AM183333 HDV7 & 72.6 & 72.4 & 64.2 & 74.0 & 74.5 & 71.9 & & 79.1 & 62.7 & 54.5 & 53.4 & 49.6 & 46.4 & 37.9 & 37.7 & 19.1 & 24.2 & 23.4 & 22.0 \\
\hline AM183330 HDV8 & 74.0 & 76.9 & 63.1 & 76.9 & 79.5 & 77.4 & 79.1 & & 65.6 & 57.4 & 54.3 & 52.0 & 46.2 & 37.2 & 39.5 & 19.5 & 22.6 & 27.4 & 20.4 \\
\hline ovDeV & 63.3 & 62.6 & 62.4 & 67.0 & 62.6 & 66.7 & 62.7 & 65.6 & & 67.0 & 57.7 & 58.9 & 51.3 & 37.2 & 36.6 & 24.5 & 23.1 & 22.3 & 21.9 \\
\hline $\mathrm{mmDeV}$ & 57.7 & 56.4 & 60.3 & 60.8 & 53.4 & 54.4 & 54.5 & 57.4 & 67.0 & & 54.6 & 55.8 & 49.7 & 39.9 & 32.7 & 22.1 & 23.7 & 22.8 & 21.5 \\
\hline MK598012 RDeV & 55.1 & 54.8 & 55.6 & 56.1 & 52.3 & 54.8 & 53.4 & 54.3 & 57.7 & 54.6 & & 64.1 & 54.7 & 36.0 & 33.0 & 19.9 & 19.4 & 20.0 & 21.8 \\
\hline $\operatorname{tg} \mathrm{DeV}$ & 51.3 & 51.5 & 54.3 & 52.8 & 50.0 & 52.0 & 49.6 & 52.0 & 58.9 & 55.8 & 64.1 & & 54.2 & 37.2 & 33.8 & 20.2 & 23.3 & 21.8 & 19.9 \\
\hline MH988742 SDeV & 48.5 & 46.2 & 49.7 & 47.2 & 45.3 & 48.7 & 46.4 & 46.2 & 51.3 & 49.7 & 54.7 & 54.2 & & 35.9 & 35.1 & 17.2 & 21.7 & 20.8 & 19.6 \\
\hline MH824555 duck DeV & 40.2 & 35.6 & 36.7 & 39.4 & 36.8 & 37.2 & 37.9 & 37.2 & 37.2 & 39.9 & 36.0 & 37.2 & 35.9 & & 29.5 & 15.3 & 16.8 & 22.3 & 16.2 \\
\hline scDeV & 35.0 & 37.1 & 35.1 & 33.2 & 38.1 & 37.1 & 37.7 & 39.5 & 36.6 & 32.7 & 33.0 & 33.8 & 35.1 & 29.5 & & 17.3 & 21.8 & 22.3 & 17.9 \\
\hline MN031240 fish DeV & 22.4 & 19.0 & 19.0 & 19.0 & 20.0 & 20.6 & 19.1 & 19.5 & 24.5 & 22.1 & 19.9 & 20.2 & 17.2 & 15.3 & 17.3 & & 15.0 & 12.5 & 14.0 \\
\hline MK962760 toad DeV & 21.5 & 24.6 & 20.6 & 23.6 & 23.7 & 22.6 & 24.2 & 22.6 & 23.1 & 23.7 & 19.4 & 23.3 & 21.7 & 16.8 & 21.8 & 15.0 & & 15.4 & 14.0 \\
\hline N031239 newt DeV & 23.6 & 25.6 & 20.0 & 23 & 24.7 & 23.3 & 23 & 27.4 & 22.3 & 22. & 20.0 & 21.8 & 20.8 & 22.3 & 22.3 & 12.5 & 15.4 & & 15.0 \\
\hline MK962759 termite DeV & 21.3 & 20.4 & 20.4 & 20.4 & 22.4 & 21.5 & 22.0 & 20.4 & 21.9 & 21.5 & 21.8 & 19.9 & 19.6 & 16.2 & 17.9 & 14.0 & 14.0 & 15.0 & \\
\hline
\end{tabular}

b

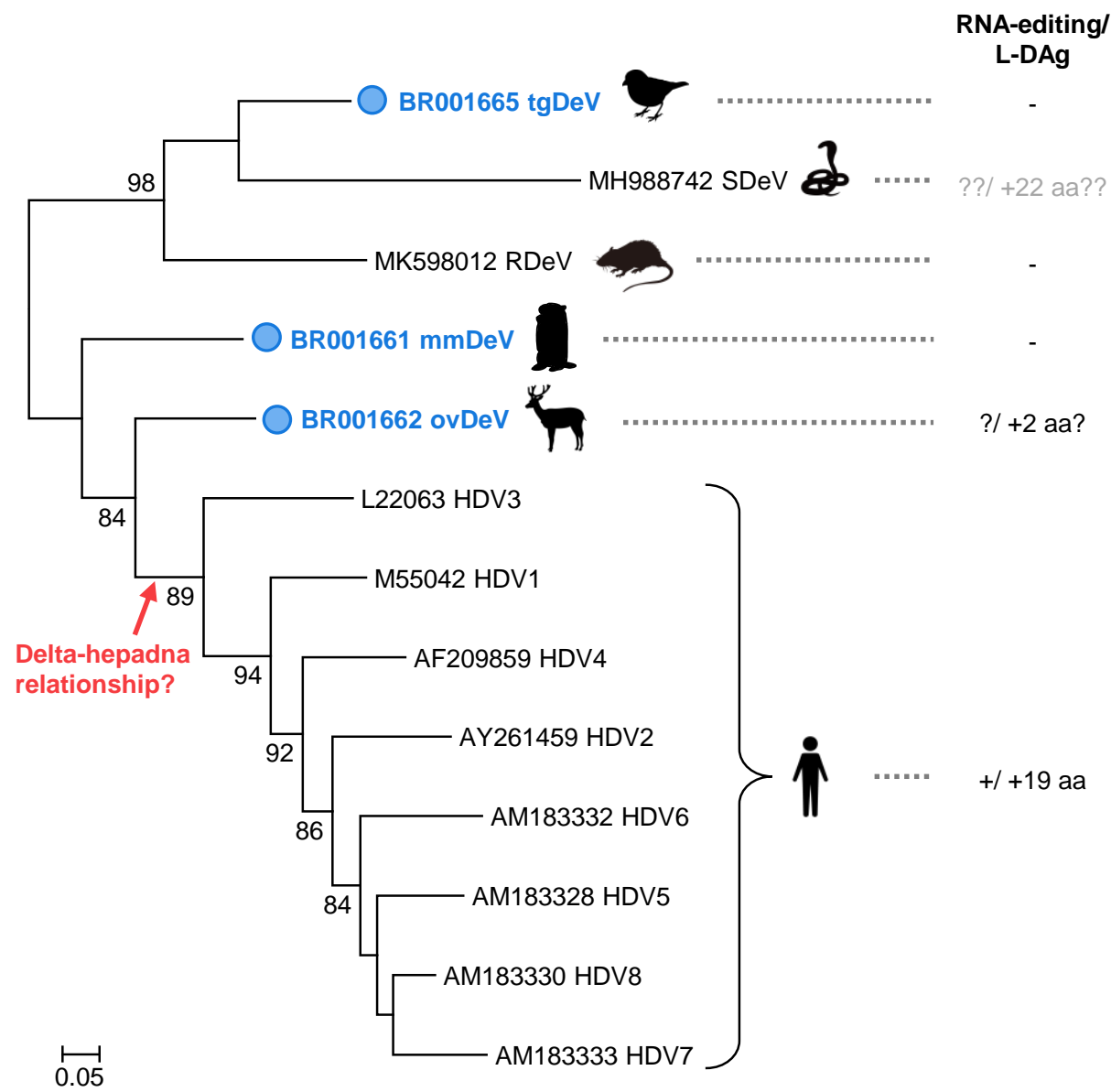

\section{Candidate helper virus}

$?$

Reptarenavirus Hartmanivirus

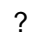

$?$

?

HBV
Identity (\%) High

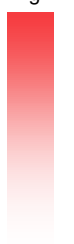

Low

HBs usage 
a

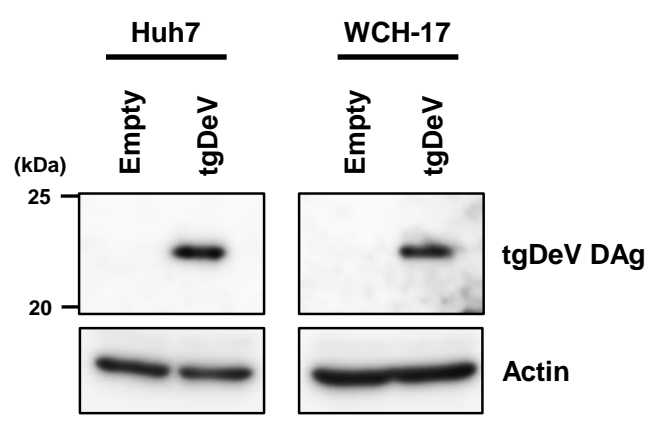

C
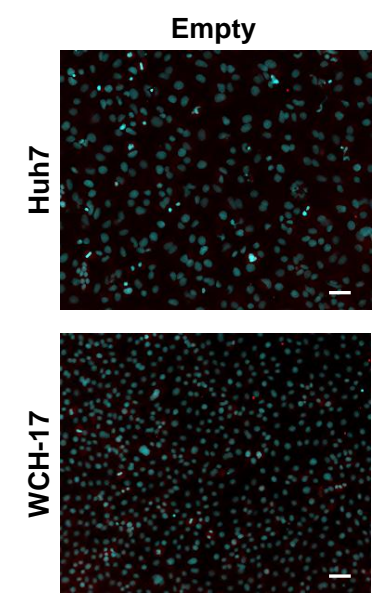

Blue: DAPI
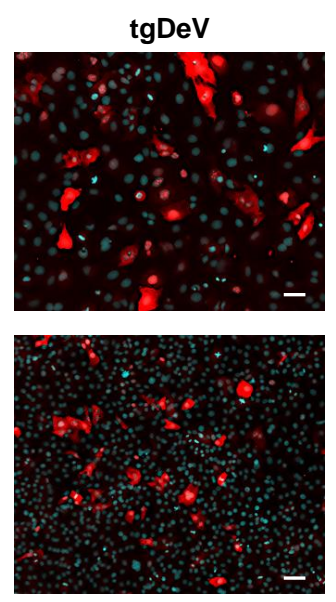

Red : tgDeV DAg

e
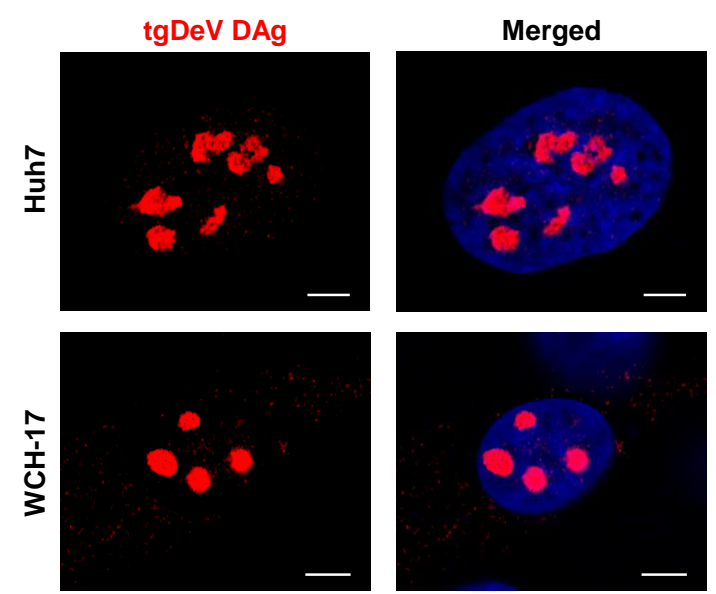

b

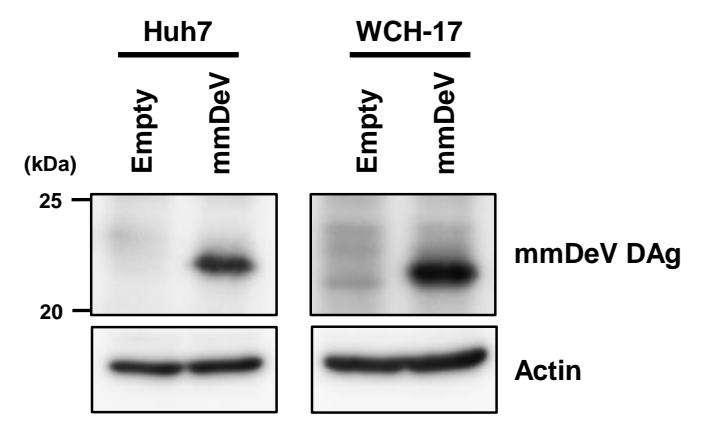

d
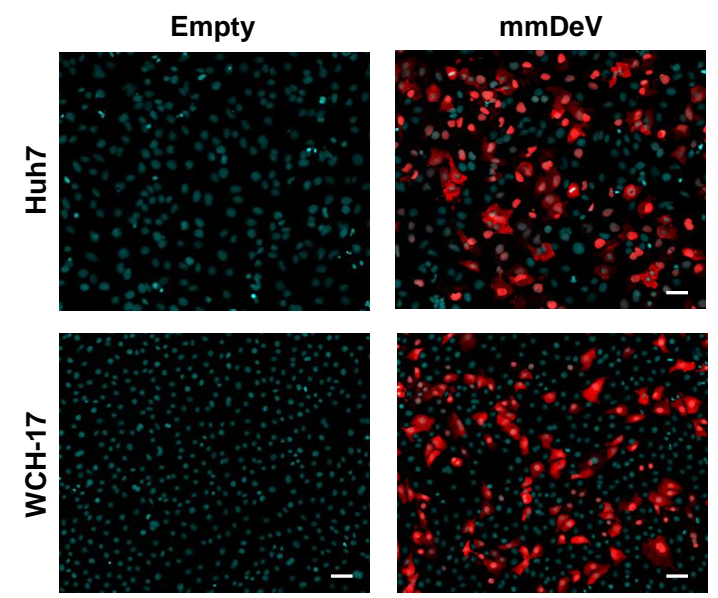

Blue: DAPI

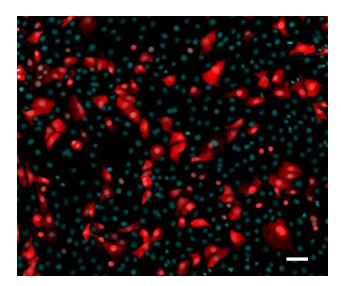

Red : mmDeVAg

f
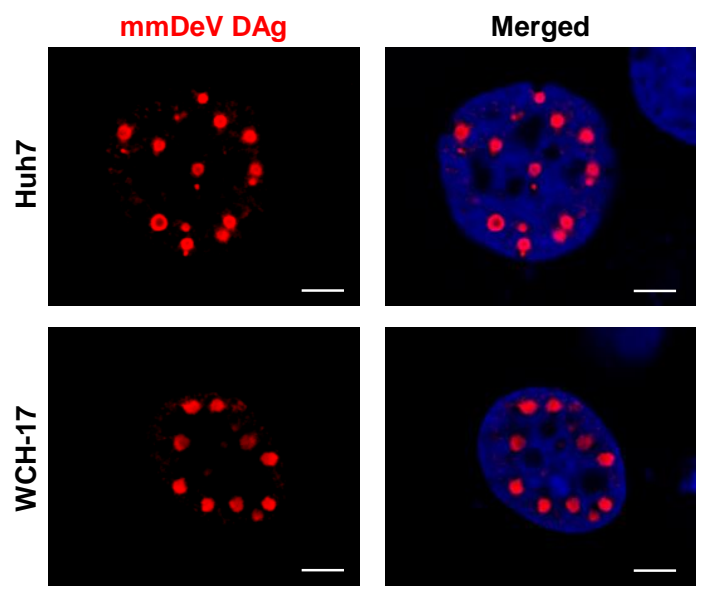
Fig. 7

a

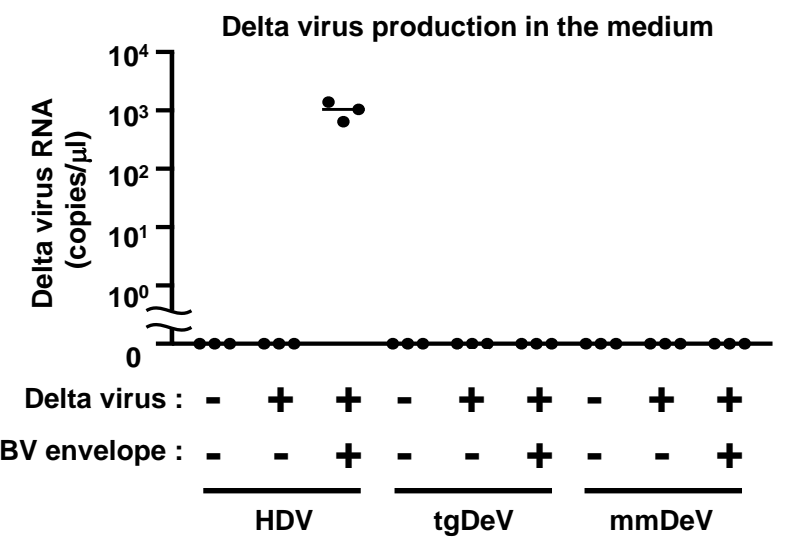

b

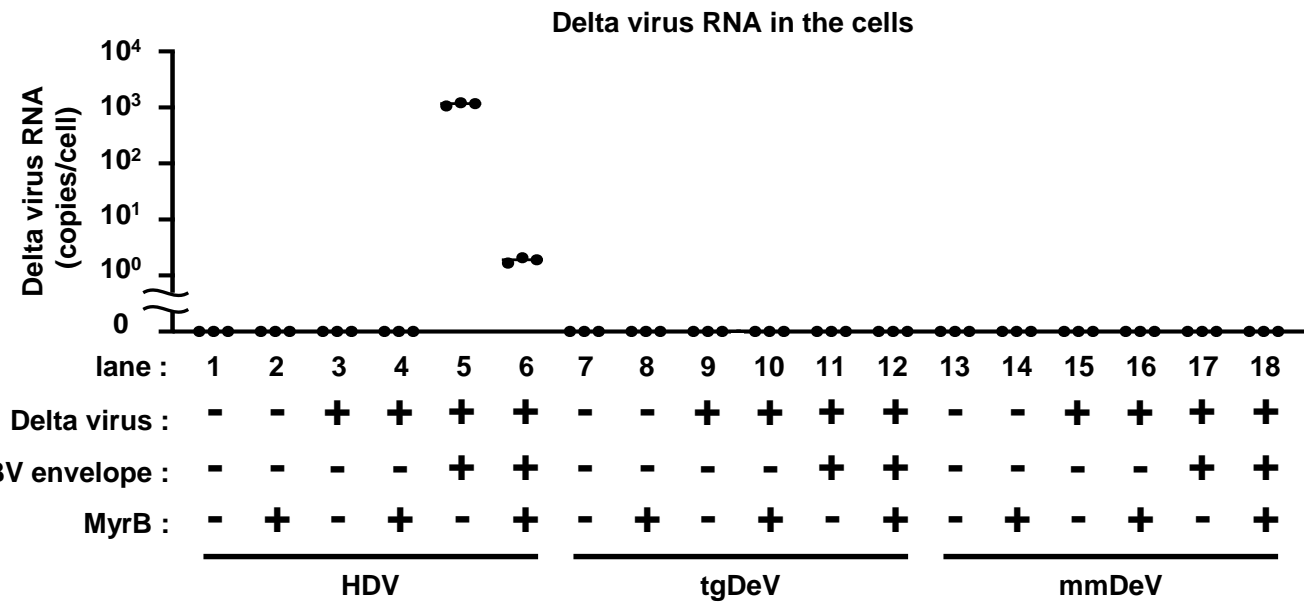

C
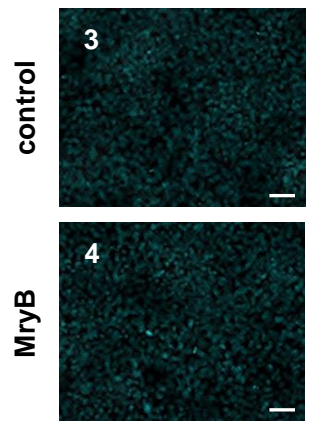

HDV
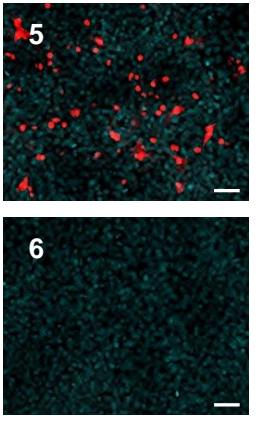
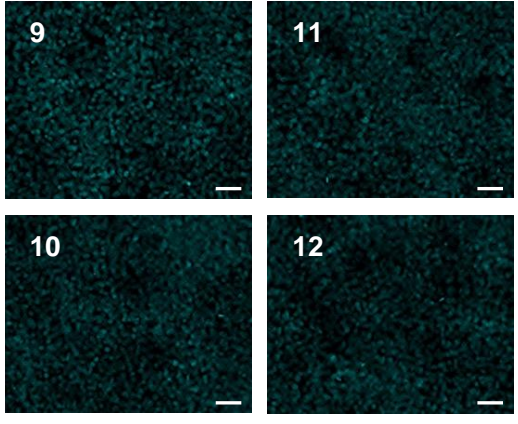

$\operatorname{tg} \mathrm{DeV}$

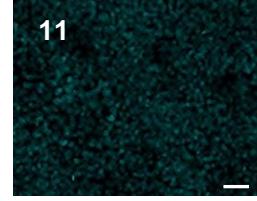

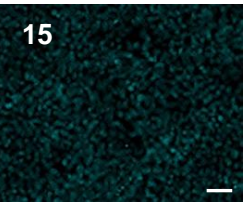

16

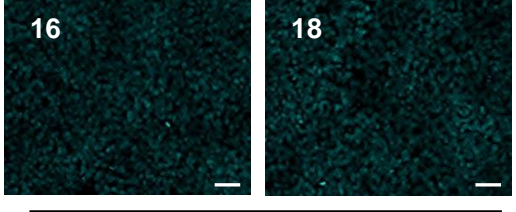

$\mathrm{mmDeV}$

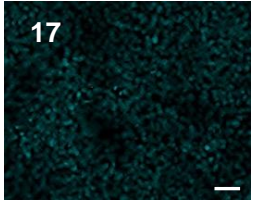

(1)

Blue: nucleus Red: DAg 